\title{
Lagrangian Averaged Stochastic Advection by Lie Transport for Fluids
}

\author{
Theodore D. Drivas ${ }^{1}$ • Darryl D. Holm² ${ }^{2}$ James-Michael Leahy ${ }^{2}$
}

Received: 29 August 2019 / Accepted: 8 January 2020 / Published online: 29 January 2020

(c) The Author(s) 2020

\begin{abstract}
We formulate a class of stochastic partial differential equations based on Kelvin's circulation theorem for ideal fluids. In these models, the velocity field is randomly transported by whitenoise vector fields, as well as by its own average over realizations of this noise. We call these systems the Lagrangian averaged stochastic advection by Lie transport (LA SALT) equations. These equations are nonlinear and non-local, in both physical and probability space. Before taking this average, the equations recover the Stochastic Advection by Lie Transport (SALT) fluid equations introduced by Holm (Proc R Soc A 471(2176):20140963, 2015). Remarkably, the introduction of the non-locality in probability space in the form of momentum transported by its own mean velocity gives rise to a closed equation for the expectation field which comprises Navier-Stokes equations with Lie-Laplacian 'dissipation'. As such, this form of non-locality provides a regularization mechanism. The formalism we develop is closely connected to the stochastic Weber velocity framework of Constantin and Iyer (Commun Pure Appl Math 61(3):330-345, 2008) in the case when the noise correlates are taken to be the constant basis vectors in $\mathbb{R}^{3}$ and, thus, the Lie-Laplacian reduces to the usual Laplacian. We extend this class of equations to allow for advected quantities to be present and affect the flow through exchange of kinetic and potential energies. The statistics of the solutions for the LA SALT fluid equations are found to be changing dynamically due to an array of intricate correlations among the physical variables. The statistical properties of the LA SALT physical variables propagate as local evolutionary equations which when spatially integrated become dynamical equations for the variances of the fluctuations. Essentially, the LA SALT theory is a non-equilibrium stochastic linear response theory for fluctuations in SALT fluids with advected quantities.
\end{abstract}

Communicated by Valerio Lucarini.

$凶$ Darryl D. Holm

d.holm@imperial.ac.uk

Theodore D. Drivas

tdrivas@math.princeton.edu

James-Michael Leahy

j.leahy@imperial.ac.uk

1 Department of Mathematics, Princeton University, Princeton, NJ 08544, USA

2 Department of Mathematics, Imperial College, London SW7 2AZ, UK 


\section{Introduction: SALT and LA SALT}

In honour of the 150th anniversary of the publication of Kelvin's circulation theorem for Euler's fluid equations of deterministic incompressible flow, Drivas and Holm [3] derived two classes of stochastic Euler fluid equations, distinguished by whether they either preserve circulation as in Holm [1], or they preserve kinetic energy, as in [4] and [5]. The paper [3] also introduced the mean field equation "in the sense of McKean" [6] for stochastic Euler fluid dynamics which preserves a statistical circulation theorem. The resulting mean field equation in paper [3] was related to the results of Constantin and Iyer [2], in which smooth solutions of Navier-Stokes are shown to be characterized by a special case of this statistical Kelvin's theorem. The present paper extends the mean field approach of [3] to include stochastic fluid equations with potential energy whose deterministic version falls in the geometric class of fluid equations studied in Holm, Marsden, and Ratiu [7]. These stochastic partial differential equation systems (SPDEs) comprise the equations of Lagrangian Averaged Stochastic Advection by Lie Transport (LA SALT) corresponding to the deterministic fluid equations treated in [7]. Besides the mean field, solutions of the LA SALT equations also contain the time-dependent statistics of the stochastic fluctuations around the mean field solutions. Thus, the LA SALT formulation may be regarded as a stochastic version of linear response theory.

\subsection{Purpose}

This paper develops a new class of fluid equations based on Stochastic Advection by Lie Transport (SALT) [1] by applying a type of Lagrangian Average (LA) which is the counterpart in probability space of the time average at fixed Lagrangian coordinate taken in the LANS-alpha turbulence model [8,9]. As was proven previously for SALT, the new set of LA SALT fluid equations with advected quantities preserves the fundamental properties of ideal fluid dynamics. These properties include Kelvin's circulation theorem and Lagrangian invariants such as enstrophy, helicity and potential vorticity [1]. These properties derive from the preserved Euler-Poincaré and Lie-Poisson geometric structures of the deterministic theory, whose history and basic mathematical elements are reviewed briefly in appendices 1 and 2 for the convenience of the reader. In the Kelvin circulation integral for the new theory (LA SALT), the Lagrangian Average in probability space manifests itself as advection of the Kelvin circulation loop by the expected fluid transport velocity. This is the primary modification made by applying LA to the SALT theory, [1,10-14]. This modification of the SALT theory allows the dynamics of the statistical properties of the solutions for the LA SALT fluid equations to be investigated directly.

One interesting consequence is that, due to the non-local nature of the equations in probability space, the expected dynamics contains terms which regularize the (expected) solution. Thus, the introduction of the LA modification of Kelvin's circulation integral bestows on the LA SALT version of the 3D Euler fluid similar solution properties to those of the incompressible Navier-Stokes equations. Moreover, in certain cases, the additional terms are strictly dissipative, and the LA SALT fluid equations may be regarded as non-conservative system of PDEs for the expected motion embedded into a larger conservative system which includes the fluctuation dynamics. From this viewpoint, the interaction dynamics of the two components of the full LA SALT system dissipates the Lagrangian invariant functions of the mean quantities by converting them into fluctuations, while preserving the total invariants. We will explore these properties both at the level of a general semidirect-product Hamiltonian system and more concretely for special cases of such systems where more details can be worked out. 
Plan of the paper We write the expected-quantity equations for LA SALT fluid dynamics with advected quantities in the semidirect-product Hamiltonian matrix form of equation (2.3) in Section 2. We then point out that the fluctuations are slaved to the expected equations in a certain sense. This slaving relation enables us to calculate the evolution equations for the local and spatially integrated variances of the fluctuations.

Section 3 begins by discussing the solution properties of vorticity dynamics and helicity conservation in the example of the LA SALT Euler equations for ideal fluids. In Sect. 3.3, we then discuss the mathematical well-posedness of the LA SALT Euler fluid equations. Theorem 1 establishes local existence for the LA SALT Euler fluid equations in Sobolev spaces in $d=2,3$, as well as global existence provided we work in two-spatial dimensions or the magnitudes of the noise correlates $\left\{\xi^{(k)}\right\}_{k}$ are sufficiently 'large' relative to the data and forces in three-dimensions. To establish these results, we take advantage of the fact the dynamics of the expectation field decouples from the fluctuations and solves a closed NavierStokes type equation with Lie-Laplacian 'dissipation' (LL NS). Theorem 2 establishes the results above for LL NS.

Section 4 illustrates the breadth of the LA SALT theory by discussing several examples. It begins by treating three simple examples which do not have advected quantities. These examples comprise the rigid body in 3D, the Burgers equation, and the Camassa-Holm equation. These first three examples reveal the additional information made available in the LA SALT theory for investigating both the linear transport equations for the fluctuation dynamics and the evolution equations for the expected physical variables, such as variances. In conclusion, we demonstrate how a more advanced example fits into the LA SALT theory. For this purpose, we formulate the 3D LA SALT incompressible stratified magnetohydrodynamics (MHD) equations and point out that MHD at this level also contains 3D Euler-Boussinesq, whose 2D case has been treated in the companion paper in this special issue [15].

\section{Main Content of the Paper}

- Stochastic Euler fluid equations which are "nonlinear in the sense of McKean" $[2,3,6,16]$ are generalised to include advected quantities.

- In certain cases, the dynamical equations for the expected values of physical variables decouple as a subsystem from the fluctuation dynamics. In the absence of advected quantities, a particular case yields an extension of the Navier-Stokes partial differential equations (PDE).

- The SPDEs for the dynamics of the fluctuations of the momentum and advected quantities are shown to be transported by the PDE solutions for the expected values.

- The statistical properties of the fluctuations are found to evolve dynamically, driven by an intricate array of correlations. Specifically, the statistical properties of the LA SALT physical variables propagate as local equations and yield dynamical variances when spatially integrated. In certain cases for which the dynamics of the fluctuations occurs by linear transport, the equations for their statistical properties form closed evolutionary PDE systems.

- Analytical conditions are found for which the LA SALT Euler fluid equations (a specific example of the LA SALT equations) are well-posed.

For the reader's convenience, the historical context and the basic mathematical elements of our present geometrical approach to fluid dynamics are briefly surveyed in Appendix 1 and 2 , respectively.

Flow Interpretation of Stochastic Advection by Lie Transport (SALT) In a paper in 2015, Holm [1] extended the Clebsch approach of [17] for deriving Euler-Poincaré equations for 
fluids with advected quantities to the case that the fluid variables undergo flows on Diff or SDiff generated via Stochastic Advection by Lie Transport (SALT). The papers $[1,18]$ derived the SALT class of stochastic continuum equations from Hamilton's principle with a stochastic advection constraint on Lagrangian fluid trajectories. Later, the stochastic constraint in [1] was derived in [10] from a corresponding two-scale decomposition of the deterministic fluid flow map using a deterministic homogenisation procedure. In [10], the fluid flow map

$$
x_{t}:=g_{t} x_{0} \in M \text { with } g_{0} x_{0}=x_{0}
$$

is decomposed into a composition of two time-dependent diffeomorphisms

$$
g_{t}=\tilde{g}_{t / \epsilon} \circ \bar{g}_{t}^{\epsilon}=\left(\mathrm{Id}+\gamma_{t / \epsilon}\right) \circ \bar{g}_{t}^{\epsilon} .
$$

The above formula factorises the flow map $g_{t}$ into the composition of two maps with different time scales $t$ and $t / \epsilon$ which are well-separated when $\epsilon \ll 1$. Let $u_{t}=\dot{g}_{t} g_{t}^{-1}$ and define $\bar{x}_{t}^{\epsilon}\left(x_{0}\right)=\bar{g}_{t}^{\epsilon} x_{0}$ to be the fluid trajectory associated with the slow time. It follows from the decomposition (1.1) and the chain rule that

$$
\left(\operatorname{Id}+\nabla_{\bar{x}_{t}^{\epsilon}} \gamma\left(\bar{x}_{t}^{\epsilon}\left(x_{0}\right), t / \epsilon\right)\right) \dot{\bar{x}}_{t}^{\epsilon}\left(x_{0}\right)=u_{t}\left(\bar{g}_{t}^{\epsilon} x_{0}+\gamma_{t / \epsilon} \bar{g}_{t}^{\epsilon} x_{0}\right)-\frac{1}{\epsilon} \frac{\partial}{\partial(t / \epsilon)} \gamma\left(\bar{x}_{t}^{\epsilon}\left(x_{0}\right), t / \epsilon\right),
$$

where $\dot{\bar{x}}_{t}^{\epsilon}\left(x_{0}\right)$ is the the time-derivative of $\bar{x}_{t}^{\epsilon}\left(x_{0}\right)$ at fixed $x_{0}$.

Deterministic multi-time homogenisation theory was used in [10] to show that $\bar{x}^{\epsilon}\left(x_{0}\right)=$ $\bar{g}^{\epsilon} x_{0}$ tends to $\bar{x}\left(x_{0}\right)=\bar{g} x_{0}$ in the limit as $\epsilon \rightarrow 0$, where $\bar{x}_{t}\left(x_{0}\right)$ is the solution of the following Itô stochastic differential equation

$$
\mathrm{d} \bar{x}_{t}\left(x_{0}\right)=\bar{u}_{t}\left(\bar{x}_{t}\left(x_{0}\right)\right) \mathrm{d} t+\sum_{k} \sigma\left(\bar{x}_{t}\left(x_{0}\right)\right) \mathrm{d} W_{t},
$$

provided two additional conditions are satisfied. First, one requires that $\partial_{t} \gamma$ has a positive Lyapunov exponent, so there exists a compact attractor and an ergodic invariant probability measure $\mu$ associated with $\partial_{t} \gamma$ arising from sensitivity to initial conditions. Second, one requires a centering condition $\int(\operatorname{Id}+\nabla \gamma)^{-1} u d \mu=0$ to hold, so the fluctuating flow velocity has zero mean when pulled back to the mean flow.

Remark 1 (The SALT theory) The SALT theory begins with the stochastic flow map $\phi_{t}$ generated by the following Stratonovich stochastic vector field equivalent to (1.2) ${ }^{1}$

$$
\mathrm{d} x_{t}(x)=u_{t}^{L}(x) \mathrm{d} t+\sum_{k=1}^{\infty} \xi^{(k)}(x) \circ \mathrm{d} W_{t}^{(k)}, \quad x \in M,
$$

1 We use the notation

$$
\mathrm{d} x_{t}(x)=b_{t}(x) \mathrm{d} t+\sum_{k} \xi^{(k)}(x) \circ \mathrm{d} W_{t}^{(k)}, \quad x \in M,
$$

to denote the stochastic vector-field associated with the stochastic flow $\phi=\left\{\phi_{s, t}\right\}_{0 \leq s \leq t}$ :

$$
\mathrm{d} \phi_{s, t}(x)=b_{t}\left(\phi_{s, t}(x)\right) \mathrm{d} t+\sum_{k} \xi^{(k)}\left(\phi_{s, t}(x)\right) \circ \mathrm{d} W_{t}, \quad \phi_{s, s}(x)=x \in M .
$$

That is to say, $\mathrm{d} x_{t}=\mathrm{d} \phi_{0, t} \circ \phi_{0, t}^{-1}$ is the stochastic analogue of the usual Eulerian vector field. For a given tensor-field $\tau$ on $M$, we use the notation $£_{\mathrm{d} x_{t}} \tau$ to denote the Lie derivative of $\tau$ along the stochastic vector field $\mathrm{d} X_{t}$. This may be defined simply as $£_{\mathrm{d} x_{t}} \tau=£_{b_{t}} \tau \mathrm{d} t+£_{\xi(k)} \tau \circ \mathrm{d} W_{t}^{(k)}$, or, perhaps, more satisfactorily, as $£_{\mathrm{d} x_{t}} \tau=\left.\mathrm{d}_{\epsilon} \phi_{t, t+\epsilon}^{*} \tau\right|_{\epsilon=0}$, which follows from the Itô-Kunita-Wentzell formula (see, e.g., [12]). This notation generalizes the definition of the deterministic Lie-derivative to the stochastic case. 
where the drift $u_{t}^{L}$ may be random and depend on the noise in (1.3).

Although the premise of the flow in equation (1.1) involves a separation of time scales, as we have discussed, the derivation of the stochastic vector field (1.2) or its equivalent (1.3) by using homogenisation in time introduces an assumption of ergodicity based on a positive Lyapunov exponent at faster time scales [10]. Stochastic computational simulations of SALT fluid flows using the stochastic vector field (1.3) can then possess broad-band temporal spectra whose ensemble statistics is non-Gaussian, rather than showing a separation of time sales. See $[13,14]$ for details.

In paper [1], the drift velocity of (1.3) was determined using the Clebsch variational approach in Hamilton's principle for fluid dynamics. This approach ensured that the Lagrangian invariants of the deterministic flow would still hold in each realization of the process (1.3). This is the SALT approach, in which the vector field (1.3) appears in the stochastic perturbation of the Euler-Poincaré equations with Lie transport noise. In applications of the SALT approach, the stationary vector fields $\xi^{(k)}(x)$ have been determined from a data analysis procedure involving numerical simulations of the stochastic equations of motion. Such a procedure has been successfully developed in $[11,13,14]$. Here, we will assume that these stationary vector fields have already been obtained from the appropriate data analysis for each given application.

Aim of the Present Paper The present paper aims to develop the following Lagrangian Average (LA) SALT class of stochastic continuum equations. Namely, we will consider stochastic flows $\left\{\Phi_{t}\right\}$ generated by stochastic vector fields of the form,

$$
\mathrm{d} X_{t}(x)=\mathbb{E}\left[u_{t}^{L}\right](x) \mathrm{d} t+\sum_{k=1}^{\infty} \xi^{(k)}(x) \circ \mathrm{d} W_{t}^{(k)}, \quad x \in M,
$$

when $\mathbb{E}\left[u_{t}^{L}\right]$ denotes the expectation of the velocity vector field $u_{t}^{L}$ with respect to the stochastic flow map $\phi_{t}$ generated by the SALT vector field in (1.3). It may be possible to interpret this choice as a further averaging at the homogenisation level, but we will leave this supposition for future work. For now, we simply adopt the LA SALT formulation implied by the stochastic vector field (1.4) and explore its dynamical consequences.

The LA SALT formulation will be closed here by enforcing preservation of the same Lagrangian invariants as in [1], but now along the flow (1.4). The resulting equations are nonlinear in the sense of McKean, i.e. there is a non-locality in probability space (an expectation) taken in the nonlinear term. As we will see, this modification introduces new regularizing terms into the equations for the expected dynamical variables.

\subsection{Kelvin Theorem Interpretation of SALT and LA SALT}

The modelling approach of Stochastic Advection by Lie Transport (SALT) [10-14] may be defined by enforcing a modification of the Kelvin theorem for deterministic fluids which replaces the transport velocity of the circulation loop $u_{t}^{L}$ in the deterministic Kelvin theorem by a Stratonovich stochastic vector field $\mathrm{d} x_{t}$ whose drift velocity is the same as the circulation loop velocity $u_{t}^{L}[1,3]$. That is, for a given smooth manifold $M$ :

$$
\oint_{C\left(u_{t}^{L}\right)} u_{t} \cdot d x \rightarrow \oint_{C\left(\mathrm{~d} x_{t}\right)} u_{t} \cdot d x
$$

where $\mathrm{d} x_{t}$ is the SALT stochastic vector field in (1.3). In the transition to the SALT Kelvin circulation integral in (1.5), the notation $C\left(u_{t}^{L}\right)\left(\right.$ resp. $\left.C\left(\mathrm{~d} x_{t}\right)\right)$ is used to denote that the 
closed material loop at time $t$ that is moving along the flow associated with $u^{L}$ (resp. $\mathrm{d} x_{t}$ ) after having started at time $t=0$. In particular, we have $C\left(\mathrm{~d} x_{t}\right)=\phi_{t}(C)$, which is a family of loops moving with the SALT flow $\phi_{t}$ at time $t$.

For example, in the Euler fluid case the proof of the modified stochastic Kelvin theorem for SALT [12] is given by

$$
\begin{aligned}
\mathrm{d} \oint_{\phi_{t}(C)} u_{t} \cdot d x & =\oint_{C} \mathrm{~d}\left(\phi_{t}^{*}\left(u_{0} \cdot d x_{0}\right)\right) \\
& =\oint_{C} \phi_{t}^{*}\left[\mathrm{~d} u_{0} \cdot d x_{0}+£_{\mathrm{d} x_{0}}\left(u_{0} \cdot d x_{0}\right)\right] \\
& =\oint_{\phi_{t}(C)}\left[\mathrm{d} u_{t} \cdot d x+£_{\mathrm{d} x_{t}}\left(u_{t} \cdot d x\right)\right] \\
& =-\oint_{\phi_{t}(C)} \nabla \mathrm{d} p_{t} \cdot d x=0,
\end{aligned}
$$

where $\phi_{t}^{*}$ is the pull-back of the flow $\phi_{t}, £_{\mathrm{d} x_{t}}$ is the Lie derivative along the stochastic vector field $\mathrm{d} x_{t}$ which generates the flow $\phi_{t}$, and $\mathrm{d} p_{t}$ is the pressure semimartingale for the SALT Euler fluid equation [19]

$$
\mathrm{d} u_{t} \cdot d x+£_{\mathrm{d} x_{t}}\left(u_{t} \cdot d x\right)=-\nabla \mathrm{d} p_{t} \cdot d x \quad \text { where } \operatorname{div} u_{t}=0 .
$$

We refer the reader to [12] for a rigorous discussion of the calculation in (1.6) and its association with Newton's Force Law for stochastic fluids. For more discussion of the emergence of Lie derivatives in the proof of Kelvin's circulation theorem as sketched in (1.9) for fluid flow, one may also refer to [7] in the deterministic case.

The same stochastic transport velocity $\mathrm{d} x_{t}$ in (1.3) which transports the circulation loop also advects the Lagrangian parcels in the SALT theory. The Lagrangian parcels may carry advected quantities $a$, such as heat, mass and magnetic field lines, by Lie transport along with the flow, as

$$
\mathrm{d} a+\mathfrak{f}_{\mathrm{d} x_{t}} a=0,
$$

where $\mathfrak{d}_{\mathrm{d} x_{t}} a$ is the Lie derivative of a tensor field $a$ with respect to the vector field $\mathrm{d} x_{t}$ in equation (1.3). That is, an advected tensor-field $a$ satisfies

$$
\mathrm{d}\left(\phi_{t}^{*} a(t, x)\right)=\phi_{t}^{*}\left(\mathrm{~d} a(t, x)+\mathcal{L}_{\mathrm{d} x_{t}} a(t, x)\right)=0, \quad \text { a.s. }
$$

where $\phi_{t}^{*} a=a_{0}$ is the pull-back of $a$ by the map $\phi_{t}$. Formula (1.7) defines advection as "invariance under the SALT flow". We refer the reader to [12] for more details about stochastic advection.

In this paper, we modify the SALT approach to stochastic fluid dynamics by replacing the SALT map $\phi_{t}$ by the LA SALT map $\Phi_{t}$. Correspondingly, we replace the Eulerian vector field $\mathrm{d} x_{t}(x)$ in (1.3) by the vector field $\mathrm{d} X_{t}(x)$ in (1.4). Because the drift velocity will be replaced by the expected velocity, this replacement is reminiscent of the McKean-Vlasov mean field approach for finite dimensional stochastic flow, which replaces the velocities for an interacting particle system by their empirical mean [20].

The LA SALT approach modifies the SALT Kelvin circulation in (1.5) by replacing the drift velocity in the stochastic transport loop velocity in (1.3) by its expectation, plus the same noise as in SALT. Namely, [1,3]

$$
\oint_{C\left(\mathrm{~d} x_{t}\right)} u_{t} \cdot d x \rightarrow \oint_{C\left(\mathrm{~d} X_{t}\right)} u_{t} \cdot d x,
$$


where the stochastic vector field $\mathrm{d} X_{t}$ is given in (1.4). Since the expectation in (1.4) refers to the transport velocity $u_{t}^{L}$ of the Lagrangian loop in Kelvin's theorem, we regard this process as a probabilistic type of Lagrangian Average (LA) which is the counterpart of the time average at fixed Lagrangian coordinate taken in the LANS-alpha turbulence model $[8,9]$.

For example, in the Euler fluid case the modified Kelvin theorem reads (cf. equation (1.6)),

$$
\mathrm{d} \oint_{C\left(\mathrm{~d} X_{t}\right)} u_{t} \cdot d x=\oint_{C\left(\mathrm{~d} X_{t}\right)}\left[\mathrm{d} u_{t} \cdot d x+\mathfrak{d}_{\mathrm{d} X_{t}}\left(u_{t} \cdot d x\right)\right]=0,
$$

where $£_{\mathrm{d} X_{t}}\left(u_{t} \cdot d x\right)$ denotes the Lie derivative of the 1 -form $u_{t} \cdot d x$ with respect to the vector field $\mathrm{d} X_{t}$ given in equation (1.4).

LA SALT Euler Equations Evaluating equation (1.9) implies the following stochastic Euler fluid motion equation in Stratonovich form

$$
\mathrm{d} u_{t} \cdot d x+\mathbf{P}^{\mathbb{E}} \mathfrak{E}_{\mathrm{d} X_{t}}\left(u_{t} \cdot d x\right)=0,
$$

where the projector $\mathbf{P}^{\mathbb{E}}$ keeps the expectation of the transport velocity $\left(\mathbb{E}\left[u_{t}^{L}\right]\right.$ ) divergencefree. Requiring the expected transport velocity to be divergence-free and allowing the fluctuation field to be compressible will turn out to endow the LA SALT equations with the same Lie-Poisson Hamiltonian structure as that of both the SALT equations and their deterministic counterparts. Moreover, applying only the projector $\mathbf{P}^{\mathbb{E}}$ in equation (1.10) is the minimal assumption needed for the flow generated by $d X_{t}$ to be volume preserving, since our $\xi$ 's are taken to be divergence-free.

This point will be detailed in Sect. 2. The equivalent Itô form of the Stratonovich equation (1.10) is

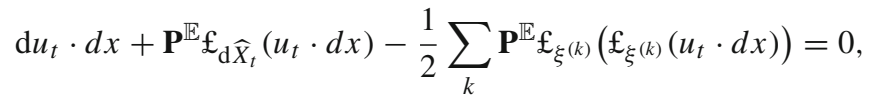

with Itô vector field $\mathrm{d} \widehat{X}_{t}$ given by

$$
\mathrm{d} \widehat{X}_{t}(x)=\mathbb{E}\left[u_{t}^{L}\right](x) \mathrm{d} t+\sum_{k} \xi^{(k)}(x) \mathrm{d} W_{t}^{(k)} .
$$

Equations are said to be "nonlinear in the sense of McKean" $[2,6,16]$ when the drift term involves the expected value of the flow it drives, as occurs in the pair of stochastic differential equations for the stochastic Euler fluid in (1.11) and (1.12). This perspective is also adopted by [16] in the special case of incompressible fluid and is used there as a route towards obtained a representation theorem for solutions of the deterministic incompressible Navier-Stokes equations.

The expectation of the Itô form of the Euler fluid motion equation (1.11) yields a motion equation with additional terms. This is the Navier-Stokes equation with Lie-Laplacian 'dissipation' [3],

$$
\partial_{t} \mathbb{E}\left[u_{t}\right] \cdot d x+\mathbf{P} £_{\mathbb{E}\left[u_{t}^{L}\right]}\left(\mathbb{E}\left[u_{t}\right] \cdot d x\right)-\frac{1}{2} \sum_{k} \mathbf{P} £_{\xi(k)}\left(£_{\xi(k)}\left(\mathbb{E}\left[u_{t}\right] \cdot d x\right)=0,\right.
$$

where $\mathbf{P}$ denotes the standard Leray projector of the vector coefficients onto their divergencefree part. Note that, in general, the Lie-Laplacian operator is not dissipative. However, it is a uniformly elliptic operator which has the effect of regularizing the solution, provided the noise correlates satisfy certain minor conditions. The LLNS equation (1.13) 
reduces to the Navier-Stokes equation for the special choice of the noise correlates $\xi^{(k)}=$ $\left\{(1,0,0)^{T},(0,1,0)^{T},(0,0,1)^{T}\right\}$, for $k=1,2,3$.

The LA SALT equations (1.11) and (1.13) first arose, along with additional noisy and viscous terms, in Lemma 3 of [3] where they were shown to govern the dynamics of the so-called 'stochastic Weber velocity'. Constantin and Iyer [2] also used stochastic fluid motion equations of the type (1.11)-(1.12) with constant $\xi^{(k)}$, where $k=1,2,3$, as a tool to represent solutions to the incompressible Navier-Stokes equations as an average over a stochastic process. In particular, in the special case of constant $\xi$ 's, the following statistical Kelvin theorem was derived in [2],

$$
v_{t}=\mathbb{E} u_{t}, \quad \oint_{C} v_{t} \cdot \mathrm{d} x=\mathbb{E} \oint_{A_{t}(C)} v_{0} \cdot \mathrm{d} x,
$$

in notation where $A_{t}=\Phi_{t}^{-1}$ and the map $\Phi_{t}$ is the flow (1.4) defined implicitly by the solution $v_{t}$. The statement that (1.14) holds for all rectifiable loops $C$ also characterizes the solution of the more general class of Lie-Laplacian Navier-Stokes (LLNS) equations in (1.13), as discussed in Drivas and Holm [3]. For further discussion of stochastic circulation and the Hamiltonian structure of the Navier-Stokes equation, see [21,22].

In summary, the LA SALT Euler fluid equations in (1.10) introduce a type of Lagrangian averaging, obtained by taking the expectation of the loop velocity $u_{t}^{L}$ in (1.12) as the velocity of the Lagrangian parcels. The LA SALT loop velocity in the modified Kelvin theorem in (1.9) will also be the transport velocity for advected quantities, when those quantities will be included in the dynamics. Thus, we may also derive LA SALT versions of compressible, adiabatic fluid dynamics, magnetohydrodynamics (MHD), etc. As mentioned earlier, taking the expectation of the Lagrangian transport velocity in the Kelvin theorem for LA SALT is analogous to taking the temporal average at fixed Lagrangian coordinate of the transport velocity for the Navier-Stokes equation to obtain the LANS-alpha equation [8,9,23-25]. The corresponding expected-quantity equations produce a Lie-Laplacian version of the NavierStokes equation.

\section{General Case of LA SALT Theories}

This section discusses the general theory of Lagrangian Averaged (LA) SALT, which is fundamentally based on Euler-Poincaré equations obtained from Hamilton's principle with symmetry-reduced Lagrangians and the Lie-Poisson Hamiltonian structures of these equations. We refer the reader to Appendix 2 for the definitions needed in the geometric setting for fluid dynamics.

Probabilistic Setting Let $\left(\Omega, \mathcal{F}, \mathbb{F}=\left\{\mathcal{F}_{t}\right\}_{t \geq 0}, \mathbb{P}\right)$ be a filtered probability space satisfying the usual conditions of right-continuity and completeness. We assume the filtered probability space supports a sequence of $\left\{W_{t}^{(k)}\right\}_{k \in \mathbb{N}, t \geq 0}$ of independent $\mathbb{R}$-valued $\mathbb{F}$-adapted Wiener process. Throughout the paper, the symbol $\xi=\left\{\xi^{(k)}\right\}_{k \in \mathbb{N}} \subset \mathfrak{X}$ will denote a collection of time-independent, deterministic, and divergence-free vector fields (i.e., $\operatorname{div}_{g} \xi^{(k)}=0$ ).

We refer the reader to [26] for more information about the basics of stochastic analysis and continuous martingale theory, to [27] for more information about stochastic integration in Hilbert spaces and stochastic partial differential equations (SPDEs), and finally to [28-30] for more information about SPDEs on manifolds.

In Sect. $\$ 2, \S 3.1$ and $\S 3.2$, we will work on arbitrary Riemannian manifolds and assume whatever regularity and conditions are needed for the relations we calculate to hold properly. 
For the concrete example of the LA SALT Euler fluid equations in Sect. § 3.3, however, we will provide a rigorous interpretation of the LA SALT equation as an SPDE on the torus. In particular, we will specify the precise regularity required of $\xi$ and the other data in $\S 3.3$ to achieve wellposedness in a suitable sense.

\subsection{Euler-Poincaré and Lie-Poisson forms of the LA SALT equations}

To take these introductory remarks further, we make the replacement (1.8) in the EulerPoincaré equation from which the SALT Kelvin circulation theorem arose in Stratonovich form, in [1]. That is, we keep the same physical class of reduced Lagrangians $\ell\left(u^{L}, a\right)$ as was treated for the SALT Euler-Poincaré variational principle in [1].

Let $\ell=\ell\left(u^{L}, a\right): \mathfrak{X} \times V \rightarrow \mathbb{R}$ be the Lagrangian. We assume that the Lagrangian possesses Gateaux derivatives $\frac{\delta \ell}{\delta u^{L}}: \mathfrak{X} \times V \rightarrow \mathfrak{X}^{*}$ and $\frac{\delta \ell}{\delta a}: \mathfrak{X} \times V \rightarrow V^{*}$ defined by

$$
\begin{aligned}
& \lim _{\varepsilon \rightarrow 0} \ell(u+\varepsilon \delta u, a)=\left\langle\frac{\delta \ell}{\delta u^{L}}, \delta u\right\rangle_{\mathfrak{X}}, \quad \forall u, \delta u \in \mathfrak{X}, \forall a \in V \\
& \lim _{\varepsilon \rightarrow 0} \ell(u, a+\varepsilon \delta a)=\left\langle\frac{\delta \ell}{\delta a}, \delta a\right\rangle_{V}, \quad \forall u \in \mathfrak{X}, \forall a, \delta a \in V .
\end{aligned}
$$

We also assume the Lagrangian is hyperregular; that is, the map $\delta \ell / \delta u^{L}(\cdot, a): \mathfrak{X} \rightarrow \mathfrak{X}^{*}$ is invertible for every $a \in V$. The SALT equations for $\mathbb{F}$-adapted $u^{L}: \Omega \times \mathbb{R}_{+} \rightarrow \mathfrak{X}$, $a: \Omega \times \mathbb{R}_{+} \rightarrow V$, and $\frac{\delta \ell}{\delta u^{L}}=\frac{\delta \ell}{\delta u^{L}}\left(u^{L}, a\right): \Omega \times \mathbb{R}_{+} \rightarrow \mathfrak{X}^{*}$, introduced in [1] read

$$
\begin{gathered}
\mathrm{d} \frac{\delta \ell}{\delta u^{L}}+\mathfrak{f}_{\mathrm{d} x_{t}} \frac{\delta \ell}{\delta u^{L}} \stackrel{\mathfrak{X}^{*}}{=} \frac{\delta \ell}{\delta a} \diamond a \mathrm{~d} t \text { and } \mathrm{d} a+\mathfrak{£}_{\mathrm{d} x_{t}} a \stackrel{V}{=} 0, \\
\text { with } \mathrm{d} x_{t}=u_{t}^{L} \mathrm{~d} t+\sum_{k=1}^{\infty} \xi^{(k)} \circ \mathrm{d} W_{t}^{(k)} .
\end{gathered}
$$

In this paper, we replace the stochastic transport vector field $\mathrm{d} x_{t}$ in (2.2) with $\mathrm{d} X_{t}$ in (2.3) to consider

$$
\begin{gathered}
\mathrm{d} \frac{\delta \ell}{\delta u^{L}}+\mathfrak{f}_{\mathrm{d} X_{t}} \frac{\delta \ell}{\delta u^{L}} \stackrel{\mathfrak{X}^{*}}{=} \mathbb{E}\left[\frac{\delta \ell}{\delta a}\right] \diamond a \mathrm{~d} t \text { and } \mathrm{d} a+\mathfrak{d}_{\mathrm{d} X_{t}} a \stackrel{V}{=} 0 \\
\text { with } \mathrm{d} X_{t}=\mathbb{E}\left[u_{t}^{L}\right] \mathrm{d} t+\sum_{k} \xi^{(k)} \circ d W_{t}^{(k)}
\end{gathered}
$$

The above equations comprise the class of LA SALT theories which we consider in the present paper.

Let $D=\rho d V \in$ Den denote the mass density of the fluid, which is one of the advected variables:

$$
d D+£_{d X_{t}} D \stackrel{\Omega^{d}}{=} 0
$$

Upon using the product rule, we derive the Kelvin-Noether form (see Corollary 6.3 in [7]) of the LA SALT motion equation in (2.3), namely,

$$
\mathrm{d}\left(\frac{1}{D} \frac{\delta \ell}{\delta u^{L}}\right)+\mathfrak{f}_{\mathrm{d} X_{t}}\left(\frac{1}{D} \frac{\delta \ell}{\delta u^{L}}\right) \stackrel{\Omega^{1}}{=} \frac{1}{D} \mathbb{E}\left[\frac{\delta \ell}{\delta a}\right] \diamond a \mathrm{~d} t .
$$


The Kelvin-Noether Circulation theorem for LA SALT follows from the Kunita-ItôWentzell formula (see Theorem 3.3 of [12]):

$$
\mathrm{d} \oint_{C\left(d X_{t}\right)} \frac{1}{D} \frac{\delta \ell}{\delta u^{L}}=\oint_{C\left(d X_{t}\right)} \frac{1}{D} \mathbb{E}\left[\frac{\delta \ell}{\delta a}\right] \diamond a,
$$

where $C\left(d X_{t}\right)=\phi_{t}(C)$ is any closed-material loop $C$ in $M$ moving with the velocity $d X_{t}$.

Define the notation

$$
v=\left(\frac{1}{D} \frac{\delta \ell}{\delta u^{L}}\right)^{\#}
$$

Then using the formula for $\mathrm{ad}^{\dagger}$ given in (B.2), the condition $\operatorname{div}_{g} \xi^{(k)}=0$, and the definition of $\hat{\diamond}$ given in (B.6) we get

$$
d v+\operatorname{ad}_{d X_{t}}^{\dagger} v+\operatorname{div}_{g}\left(\mathbb{E}\left[u_{t}^{L}\right]\right) v \mathrm{~d} t \stackrel{\mathfrak{x}}{=} \mathbb{E}\left[\frac{\delta \ell}{\delta a}\right] \hat{\diamond} \frac{a}{\rho} \mathrm{d} t .
$$

Legendre Transform to the Hamiltonian Side The Legendre transform from the Lagrangian side to the Hamiltonian side for SALT in [1] is given by

$$
\mu=\frac{\delta \ell}{\delta u^{L}} \text { and } \mathrm{d} h(\mu, a)=\left\langle\mu, \mathrm{d} x_{t}\right\rangle_{\mathfrak{X}}-\ell\left(u^{L}, a\right) \mathrm{d} t,
$$

where $\mathrm{d} x_{t}$ is given in equation (1.3). By introducing the deterministic Hamiltonian

$$
H(\mu, a):=\left\langle\mu, u^{L}\right\rangle_{\mathfrak{X}}-\ell\left(u^{L}, a\right),
$$

we may alternatively express the above as

$$
\mathrm{d} h(\mu, a)=H(\mu, a) d t+\sum_{k}\left\langle\mu, \xi^{(k)}\right\rangle_{\mathfrak{X}} \circ \mathrm{d} W_{t}^{(k)} .
$$

Note that $\delta h / \delta u^{L}=0$ and $\delta h / \delta a=\delta H / \delta a$ by definition. Taking variations of $h(\mu, a)$ yields

$$
\begin{aligned}
\delta h(\mu, a) & =\left\langle\delta \mu, \frac{\delta h}{\delta \mu}\right\rangle_{\mathfrak{X}}+\left\langle\frac{\delta h}{\delta a}, \delta a\right\rangle_{V} \\
& =\left\langle\delta \mu, \mathrm{d} x_{t}\right\rangle_{\mathfrak{X}}-\left\langle\frac{\delta \ell}{\delta a}, \delta a\right\rangle_{V}+\left\langle\mu-\frac{\delta \ell}{\delta u^{L}}, \delta u^{L}\right\rangle_{\mathfrak{X}}
\end{aligned}
$$

Then, upon identifying corresponding terms, one verifies the fibre derivative $\mu=\delta \ell / \delta u^{L}$ and finds the following variational derivatives of the SALT Hamiltonian,

$$
\mathrm{d} \frac{\delta h}{\delta \mu}=\mathrm{d} x_{t}=\frac{\delta H}{\delta \mu} \mathrm{d} t+\sum_{k} \xi^{(k)} \circ \mathrm{d} W_{t}^{(k)} \text { and } \frac{\delta H}{\delta a}=-\frac{\delta \ell}{\delta a} .
$$

At this point, we take expectations of the terms in (2.6) which pass from the SALT equations in (2.2) to the LA SALT equations in (2.3). Specifically, defining for LA SALT

$$
\mu=\frac{\delta \ell}{\delta u^{L}} \text { and } \mathrm{d} h(\mu, a)=\left\langle\mu, \mathrm{d} X_{t}\right\rangle_{\mathfrak{X}}-\ell\left(u^{L}, a\right) \mathrm{d} t
$$

we obtain the Hamiltonian formulation of the LA SALT equations from the Lagrangian formulation above

$$
\mathrm{d} \frac{\delta h}{\delta \mu}=\mathrm{d} X_{t}=\mathbb{E}\left[\frac{\delta H}{\delta \mu}\right] \mathrm{d} t+\sum_{k} \xi^{(k)} \circ \mathrm{d} W_{t}^{(k)} \text { and } \mathbb{E}\left[\frac{\delta H}{\delta a}\right]=-\mathbb{E}\left[\frac{\delta \ell}{\delta a}\right],
$$


where $H$ is again defined by (2.5). Taking the expectation then transforms the LA SALT equations (2.3) in Euler-Poincaré into Hamiltonian form with a Lie-Poisson matrix operator. Thus, the Stratonovich version of the SDP-LPB for the SALT Hamiltonian formulation yields the LA SALT equations in (2.3) as

$$
\mathrm{d}\left[\begin{array}{l}
\mu \\
a
\end{array}\right]=-\left[\begin{array}{c}
\mathfrak{f}_{(\cdot)} \mu(\cdot) \diamond a \\
\mathfrak{E}_{(\cdot)} a
\end{array}\right]\left[\begin{array}{c}
\mathbb{E}[\delta H / \delta \mu] \mathrm{d} t+\sum_{k} \xi^{(k)} \circ \mathrm{d} W_{t}^{(k)} \\
\mathbb{E}[\delta H / \delta a] \mathrm{d} t
\end{array}\right] .
$$

The definition of the diamond operator $(\diamond)$ (B.5) ensures that the Lie-Poisson matrix operator is skew-symmetric under integration by parts in $L^{2}$ pairing. The modification of the Hamiltonian form of the SALT equations to obtain the LA SALT equations in (2.7) replaces the variational derivative of the Hamiltonian with respect to momentum and to the advected variable by their expected values. This modification preserves the Hamiltonian matrix operator in both the deterministic and SALT formulations and makes that operator available for exploring the solution behaviour for LA SALT, as we discuss below in a combination of theorems and illustrative examples. Essentially, we will discover below that the LA SALT theory is a nonequilibrium stochastic linear response theory for fluctuations in SALT fluids with advected quantities.

Remark 2 We will show below that the expectation of the system (2.7) results in a closed dynamical system, when the expected variational derivatives $\delta H / \delta \mu$ and $\delta H / \delta a$ are linear in the variables $\mu$ and $a$, after one has accounted for the constraints (e.g. incompressibility). As we shall see, upon regarding SALT as the 'mother theory', LA SALT can be regarded as a first-order cumulant discard closure for SALT and therefore it can be characterized as a type of linear response theory, particularly because its dynamics involves both fluctuations and dissipation. We will investigate several examples of this situation in the remainder of the paper.

Casimirs The LA SALT system (2.7) undergoes stochastic coadjoint motion. That is, for any given functional $C: \mathfrak{X}^{*} \times V^{*} \rightarrow \mathbb{R}$, the LA SALT dynamics of $C[\mu, a]$ is given by

$$
\begin{aligned}
& \mathrm{d} C[\mu, a]=\left\langle d \mu, \frac{\delta C}{\delta \mu}\right\rangle_{\mathfrak{X}}+\left\langle\frac{\delta C}{\delta a}, d a\right\rangle_{V}=\left\langle d \mu, \frac{\delta C}{\delta \mu}\right\rangle_{\mathfrak{X}}+\left\langle d a, \frac{\delta C}{\delta a}\right\rangle_{V^{*}}
\end{aligned}
$$

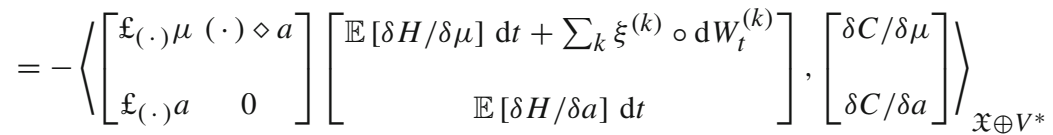

A functional $C[\mu, a]$ whose variational derivatives $[\delta C / \delta \mu, \delta C / \delta a]^{T}$ comprise a null eigenvector of the Hamiltonian matrix operator in (2.8) is called a Casimir functional for that Lie-Poisson system. Casimir functionals satisfy $\mathrm{d} C[\mu, a]=0$, so that $C\left[\mu_{t}, a_{t}\right]=$ $C\left[\mu_{0}, a_{0}\right]$ for any Hamiltonian $H[\mu, a]$. By having preserved the Lie-Poisson structure of the deterministic Hamiltonian fluid equations in formulating the LA SALT system (2.7), one has preserved the Casimir conserved quantities for the original deterministic Lie-Poisson fluid dynamics. In turn, one has also preserved the expectations of the Casimirs, since for them $\mathbb{E}\left[C\left[\mu_{t}, a_{t}\right]\right]=\mathbb{E}\left[C\left[\mu_{0}, a_{0}\right]\right]$.

Thus, equation (2.8) for LA SALT encapsulates all three of the stages in the approximations of fluid dynamics which we have been discussing. The SALT formulation in [1] emerges when the expectations are not taken in the first term of the product in the integrand. The historical 
deterministic formulation discussed in the Introduction emerges when the $\xi^{(k)}$ also vanish in that term. Since the Casimirs are defined as null vectors of the same Hamiltonian operator in each case, they persist in all three stages of deterministic, SALT and LA SALT fluid dynamics.

In summary, because the LA SALT modification of the SALT transport vector field preserves the form of the reduced Euler-Poincaré Lagrangian in (2.3) and the Lie-Poisson Hamiltonian operator in (2.7), one retains both the Kelvin circulation theorem and the conservation of Casimirs of the Lie-Poisson bracket in the LA SALT dynamics.

\subsection{Closed Dynamics of the Average}

Significant simplifications occur when the drift velocity of SALT is replaced by its expectation in LA SALT. Indeed, upon converting (2.3) to Itô-form, we find

$$
\begin{aligned}
\mathrm{d} \mu+£_{\mathbb{E}\left[\frac{\delta H}{\delta \mu}\right]} \mu \mathrm{d} t+£_{\xi(k)} \mu \mathrm{d} W_{t}^{(k)} & =\left(\frac{1}{2} \sum_{k} £_{\xi(k)}\left(£_{\xi(k)} \mu\right) \mathrm{d} t-\mathbb{E}\left[\frac{\delta H}{\delta a}\right] \diamond a\right) \mathrm{d} t \\
\mathrm{~d} a+£_{\mathbb{E}\left[\frac{\delta H}{\delta \mu}\right]} a \mathrm{~d} t+£_{\xi^{(k)}} a \mathrm{~d} W_{t}^{(k)} & =\frac{1}{2} \sum_{k} £_{\xi^{(k)}}\left(£_{\xi^{(k)}} a\right) \mathrm{d} t .
\end{aligned}
$$

Taking the expectation of (2.9) then yields

$$
\begin{aligned}
\frac{\mathrm{d}}{\mathrm{d} t} \mathbb{E}[\mu]+£_{\mathbb{E}\left[\frac{\delta H}{\delta \mu}\right]} \mathbb{E}[\mu] & =\frac{1}{2} \sum_{k} £_{\xi^{(k)}}\left(£_{\xi(k)} \mathbb{E}[v]\right)-\mathbb{E}\left[\frac{\delta H}{\delta a}\right] \diamond \mathbb{E}[a], \\
\frac{\mathrm{d}}{\mathrm{d} t} \mathbb{E}[a]+£_{\mathbb{E}\left[\frac{\delta H}{\delta \mu}\right]} \mathbb{E}[a] & =\frac{1}{2} \sum_{k} £_{\xi^{(k)}}\left(£_{\xi^{(k)}} \mathbb{E}[a]\right) .
\end{aligned}
$$

Similarly, starting from (2.4) we obtain

$$
\begin{gathered}
\partial_{t} \mathbb{E}[v]+\operatorname{ad}_{\mathbb{E}\left[\frac{\delta H}{\delta \mu}\right]}^{\dagger} \mathbb{E}[v]+\operatorname{div}_{g}\left(\mathbb{E}\left[\frac{\delta H}{\delta \mu}\right]\right) \mathbb{E}[v] \\
=\frac{1}{2} \sum_{k} \operatorname{ad}_{\xi(k)}^{\dagger}\left(\operatorname{ad}_{\xi(k)}^{\dagger} \mathbb{E}[v]\right)-\mathbb{E}\left[\frac{\delta H}{\delta a}\right] \hat{\diamond} \mathbb{E}\left[\frac{a}{\rho}\right],
\end{gathered}
$$

where $\hat{\diamond}$ is defined by (B.6). These equations provide the history of the expectations $\mathbb{E}[\mu]$, $\mathbb{E}[v]$, and $\mathbb{E}[a]$ throughout the duration of the flow. If, for example, the variations $\delta H / \delta \mu$ and $\delta H / \delta a$ are linear in $\mu$ and $a$, then the equations (2.3) (equivalently, (2.9)) are slaved to the expectations $\mathbb{E}[\mu]$ and $\mathbb{E}[a]$ as linear stochastic transport relations. We refer the reader to the numerous examples in Sect. 4 for which this is the case, particularly when the flow is divergence free.

Thus, introduction of nonlocality in the sense of McKean [6] in the LA SALT equations (2.3) has significantly simplified stochastic fluid dynamics in two ways. First, it preserves the differential structure and form of the nonlinear deterministic fluid motion and advection equations that result in promotion of Lagrangian conservation laws to our setting. Second, it introduces linear equations for the fluctuations (in many special cases, including incompressible Euler), which are stochastically driven while being transported by the expectation velocity and accelerated by forces involving expectations. 


\subsection{Fluctuation Variance Dynamics}

In this section, we will discuss the fluctuations of (2.3) and (2.4) about their average. We first define the fluctuation variables by

$$
v^{\prime}:=v-\mathbb{E}[v] \in \mathfrak{X}, \quad a^{\prime}:=a-\mathbb{E}[a] \in V
$$

The dynamics of the fluctuations are obtained by taking the difference between the Stratonovich formulations (2.3) and (2.10)

$$
\begin{aligned}
& \mathrm{d} v^{\prime}+\left[\operatorname{ad}_{\mathbb{E}\left[\frac{\delta H}{\delta \mu}\right]}^{\dagger} v^{\prime}+\operatorname{div}_{g}\left(\mathbb{E}\left[\frac{\delta H}{\delta \mu}\right]\right) v^{\prime}\right] \mathrm{d} t+£_{\xi(k)} v \circ \mathrm{d} W_{t}^{(k)} \\
& =\left[-\frac{1}{2} \sum_{k} \operatorname{ad}_{\xi(k)}^{\dagger}\left(\operatorname{ad}_{\xi(k)}^{\dagger} \mathbb{E}[v]\right)-\mathbb{E}\left[\frac{\delta H}{\delta a}\right] \tilde{\diamond}\left(\frac{a}{\rho}\right)^{\prime}\right] \mathrm{d} t, \\
& \mathrm{~d} a^{\prime}+£_{\mathbb{E}\left[\frac{\delta H}{\delta \mu}\right]} a^{\prime} \mathrm{d} t+£_{\xi(k)} a \circ \mathrm{d} W_{t}^{(k)}=-\frac{1}{2} \sum_{k} £_{\xi(k)}\left(£_{\xi(k)} \mathbb{E}[a]\right) \mathrm{d} t,
\end{aligned}
$$

where $\left(\frac{a}{\rho}\right)^{\prime}=\frac{a}{\rho}-\mathbb{E}\left[\frac{a}{\rho}\right]$. The corresponding Itô-formulation of the fluctuation dynamics, obtained, for example, by taking the difference of (2.9) and (2.10), are

$$
\begin{aligned}
\mathrm{d} v^{\prime}+ & {\left[\operatorname{ad}_{\mathbb{E}\left[\frac{\delta H}{\delta \mu}\right]}^{\dagger} v^{\prime}+\operatorname{div}_{g}\left(\mathbb{E}\left[\frac{\delta H}{\delta \mu}\right]\right) v^{\prime}\right] \mathrm{d} t+\operatorname{ad}_{\xi^{(k)}}^{\dagger} v \mathrm{~d} W_{t}^{(k)} } \\
& =\left[\frac{1}{2} \sum_{k} \operatorname{ad}_{\xi(k)}^{\dagger}\left(\operatorname{ad}_{\xi(k)}^{\dagger} v^{\prime}\right)-\mathbb{E}\left[\frac{\delta H}{\delta a}\right] \tilde{\diamond}\left(\frac{a}{\rho}\right)^{\prime}\right] \mathrm{d} t, \\
\mathrm{~d} a^{\prime}+ & £_{\mathbb{E}\left[\frac{\delta H}{\delta \mu}\right]} a^{\prime} \mathrm{d} t+£_{\xi(k)} a \mathrm{~d} W_{t}^{(k)}=\frac{1}{2} \sum_{k} £_{\xi^{(k)}}\left(£_{\xi^{(k)}} a^{\prime}\right) \mathrm{d} t .
\end{aligned}
$$

This formulation simplifies the calculations in this section. Applying the Itô-product rule pointwise, we find

$$
\begin{aligned}
& \frac{1}{2} \mathrm{~d} g\left(v^{\prime}, v^{\prime}\right)+\left[g\left(\operatorname{ad}_{\mathbb{E}\left[\frac{\delta H}{\delta \mu}\right]}^{\dagger} v^{\prime}+\operatorname{div}_{g}\left(\mathbb{E}\left[\frac{\delta H}{\delta \mu}\right]\right) v^{\prime}, v^{\prime}\right)\right] \mathrm{d} t+\sum_{k} g\left(\operatorname{ad}_{\xi^{(k)}}^{\dagger} v, v^{\prime}\right) \mathrm{d} W_{t}^{(k)} \\
& =\frac{1}{2} \sum_{k}\left[g\left(\operatorname{ad}_{\xi^{(k)}}^{\dagger}\left(\operatorname{ad}_{\xi^{(k)}}^{\dagger} v^{\prime}\right)-\mathbb{E}\left[\frac{\delta H}{\delta a}\right] \tilde{\delta}\left(\frac{a}{\rho}\right)^{\prime}, v^{\prime}\right)+g\left(\operatorname{ad}_{\xi^{(k)}}^{\dagger} v, \operatorname{ad}_{\xi^{(k)}}^{\dagger} v\right)\right] \mathrm{d} t, \\
& \frac{1}{2} \mathrm{~d} g\left(a^{\prime}, a^{\prime}\right)+g\left(£_{\mathbb{E}\left[\frac{\delta H}{\delta \mu}\right]} a^{\prime}, a^{\prime}\right) \mathrm{d} t+\sum_{k} g\left(£_{\xi(k)} a, a^{\prime}\right) \mathrm{d} W_{t}^{(k)} \\
& =\frac{1}{2} \sum_{k}\left[g\left(£_{\xi^{(k)}}\left(£_{\xi^{(k)}} a^{\prime}\right), a^{\prime}\right)+g\left(£_{\xi^{(k)}} a, £_{\xi^{(k)}} a\right)\right] \mathrm{d} t .
\end{aligned}
$$

Integrating (2.13) and using stochastic Fubini's theorem (see, e.g., [31]) yields

$$
\begin{aligned}
\frac{1}{2} \mathrm{~d}\left|v^{\prime}\right|_{L^{2}}^{2}+ & {\left[\left(\operatorname{ad}_{\mathbb{E}\left[\frac{\delta H}{\delta \mu}\right]}^{\dagger} v^{\prime}+\operatorname{div}_{g}\left(\mathbb{E}\left[\frac{\delta H}{\delta \mu}\right]\right) v^{\prime}, v^{\prime}\right)_{L^{2}}\right] \mathrm{d} t+\sum_{k}\left(\operatorname{ad}_{\xi^{(k)}}^{\dagger} v, v^{\prime}\right)_{L^{2}} \mathrm{~d} W_{t}^{(k)} } \\
& =\frac{1}{2} \sum_{k}\left[\left(\operatorname{ad}_{\xi^{(k)}}^{\dagger}\left(\operatorname{ad}_{\xi^{(k)}}^{\dagger} v^{\prime}\right)-\mathbb{E}\left[\frac{\delta H}{\delta a}\right] \tilde{\delta}\left(\frac{a}{\rho}\right)^{\prime}, v^{\prime}\right)_{L^{2}}+\left(\operatorname{ad}_{\xi^{(k)}}^{\dagger} v, \operatorname{ad}_{\xi^{(k)}}^{\dagger} v\right)_{L^{2}}\right] \mathrm{d} t,
\end{aligned}
$$




$$
\begin{aligned}
& \frac{1}{2} \mathrm{~d}\left|a^{\prime}\right|_{L^{2}}+\left(£_{\mathbb{E}\left[\frac{\delta H}{\delta \mu}\right]} a^{\prime}, a^{\prime}\right)_{L^{2}} \mathrm{~d} t+\sum_{k}\left(£_{\xi(k)} a, a^{\prime}\right)_{L^{2}} \mathrm{~d} W_{t}^{(k)} \\
& =\frac{1}{2} \sum_{k}\left[\left(£_{\xi^{(k)}}\left(£_{\xi^{(k)}} a^{\prime}\right), a^{\prime}\right)_{L^{2}}+\left(£_{\xi^{(k)}} a, £_{\xi^{(k)}} a\right)_{L^{2}}\right] \mathrm{d} t \text {. }
\end{aligned}
$$

We remark that one could have directly deduced (2.14) from (2.12) under weaker assumptions than classical solutions by appealing to Itô's formula for the square of the norm (see, [31-35]). Upon making use of equations (B.3), (B.6), (B.4), and (B.5), we find

$$
\begin{aligned}
& \frac{1}{2} \mathrm{~d}\left|v^{\prime}\right|_{L^{2}}^{2}-\left[\left(\operatorname{ad}_{v^{\prime}}^{\dagger} v^{\prime}, \mathbb{E}\left[\frac{\delta H}{\delta \mu}\right]\right)_{L^{2}}+\left\langle\mathbb{E}\left[\frac{\delta H}{\delta a}\right], \mathfrak{£}_{v}\left(\frac{a}{\rho}\right)^{\prime}\right\rangle_{V}\right] \mathrm{d} t \\
& =-\frac{1}{2} \sum_{k}\left(\left[\operatorname{ad}_{v^{\prime}}^{\dagger}\left(\operatorname{ad}_{\xi^{(k)}}^{\dagger} v^{\prime}\right)+\operatorname{ad}_{v}\left(\operatorname{ad}_{\xi^{(k)}}^{\dagger} v\right)\right], \xi^{(k)}\right)_{L^{2}} \mathrm{~d} t \\
& -\left(\operatorname{div}_{g}\left(\mathbb{E}\left[\frac{\delta H}{\delta \mu}\right]\right) v^{\prime}, v^{\prime}\right)_{L^{2}}+\sum_{k}\left(\operatorname{ad}_{v}^{\dagger} v, \xi^{(k)}\right)_{L^{2}} \mathrm{~d} W_{t}^{(k)}, \\
& \frac{1}{2} \mathrm{~d}\left|a^{\prime}\right|_{L^{2}}^{2}-\left\langle\star a^{\prime} \diamond a^{\prime}, \mathbb{E}\left[\frac{\delta H}{\delta \mu}\right]\right\rangle_{\mathfrak{X}} \mathrm{d} t-\sum_{k}\left\langle\star a^{\prime} \diamond a, \xi^{(k)}\right\rangle_{\mathfrak{X}} \mathrm{d} W_{t}^{(k)} \\
& =-\frac{1}{2} \sum_{k}\left\langle\left[\star a^{\prime} \diamond\left(\mathfrak{\xi}_{\xi^{(k)}} a^{\prime}\right)+\star £_{\xi^{(k)}} a \diamond a\right], \xi^{(k)}\right\rangle_{\mathfrak{X}} \mathrm{d} t \text {. }
\end{aligned}
$$

Finally, upon taking the expectation of (2.15), we find

$$
\begin{aligned}
& \frac{1}{2} \partial_{t} \mathbb{E}\left[\left|v^{\prime}\right|_{L^{2}}^{2}\right]-\left(\mathbb{E}\left[\operatorname{ad}_{v^{\prime}}^{\dagger} v^{\prime}\right], \mathbb{E}\left[\frac{\delta H}{\delta \mu}\right]\right)_{L^{2}}-\left\langle\mathbb{E}\left[\frac{\delta H}{\delta a}\right], \mathbb{E}\left[\mathfrak{E}_{v}\left(\frac{a}{\rho}\right)^{\prime}\right]\right\rangle_{V} \\
& =-\frac{1}{2} \sum_{k}\left(\mathbb{E}\left[\operatorname{ad}_{v^{\prime}}^{\dagger}\left(\operatorname{ad}_{\xi^{(k)}}^{\dagger} v^{\prime}\right)+\operatorname{ad}_{v}\left(\operatorname{ad}_{\xi^{(k)}}^{\dagger} v\right)\right], \xi^{(k)}\right)_{L^{2}} \\
& -\left(\mathbb{E}\left[g\left(v^{\prime}, v^{\prime}\right)\right], \operatorname{div}_{g}\left(\mathbb{E}\left[\frac{\delta H}{\delta \mu}\right]\right)\right)_{L^{2}}, \\
& \frac{1}{2} \partial_{t} \mathbb{E}\left[\left|a^{\prime}\right|_{L^{2}}^{2}\right]-\left\langle\mathbb{E}\left[\star a^{\prime} \diamond a^{\prime}\right], \mathbb{E}\left[\frac{\delta H}{\delta \mu}\right]\right\rangle_{\mathfrak{X}}=-\frac{1}{2} \sum_{k}\left\langle\mathbb{E}\left[\star a^{\prime} \diamond\left(£_{\xi^{(k)}} a^{\prime}\right)+\star £_{\xi^{(k)}} a \diamond a\right], \xi^{(k)}\right\rangle_{\mathcal{X}} .
\end{aligned}
$$

In particular, upon assuming $\operatorname{div}_{g} \mathbb{E}\left[\frac{\delta H}{\delta \mu}\right]=0$ and $\rho \equiv 1$ we obtain

$$
\begin{aligned}
\frac{1}{2} \partial_{t} \mathbb{E}\left[\left|v^{\prime}\right|_{L^{2}}^{2}\right] & -\left(\mathbb{E}\left[\operatorname{ad}_{v^{\prime}}^{\dagger} v^{\prime}\right], \mathbb{E}\left[\frac{\delta H}{\delta \mu}\right]\right)_{L^{2}}-\left\langle\mathbb{E}\left[\frac{\delta H}{\delta a}\right], \mathbb{E}\left[£_{v} a^{\prime}\right]\right\rangle_{V} \\
& =-\frac{1}{2} \sum_{k}\left(\mathbb{E}\left[\operatorname{ad}_{v}^{\dagger}\left(\operatorname{ad}_{\xi^{(k)}}^{\dagger} v^{\prime}\right)+\operatorname{ad}_{v}\left(\operatorname{ad}_{\xi^{(k)}}^{\dagger} v\right], \xi^{(k)}\right)_{L^{2}} .\right.
\end{aligned}
$$

Thus, we can see that the dynamics of the variances of the stochastic system (2.11) is driven by an intricate variety of correlations among the evolving fluctuation variables. The consequences of these very general equations can be seen more easily in examples. A special case will be the vorticity dynamics in the LA SALT Euler fluid equations we shall treat next. 


\section{An Illustrative Example: LA SALT Euler}

We now show that the LA SALT Euler equations introduced in the beginning of the paper comprise a special case of the general class of the Lie-Poisson Hamiltonian systems defined by (2.3).

For a given $p \in \Omega^{0}$ on $M$, let us define $\ell: \mathfrak{X} \times V \rightarrow \mathbb{R}$ for all $u \in \mathfrak{X}$ and $D=\rho d V \in$ $V=$ Den by

$$
\ell(u, D)=\int_{M}\left(\frac{\rho}{2} g(u, u)-p(\rho-1)\right) d V=\int_{M}\left(\frac{\rho}{2} u^{b}(u)-p(\rho-1)\right) d V .
$$

Let $V^{*}=\Omega^{0}$ and define

$$
\langle b, D\rangle_{V}:=\int_{M} b \rho d V, \quad D=\rho d V \in V, \quad b \in V^{*} .
$$

Then

$$
\mu=\frac{\delta \ell}{\delta u}=u^{\mathrm{b}} \otimes D \in \mathfrak{X}^{*} \text { and } \frac{\delta \ell}{\delta D}=-\frac{\delta H}{\delta D}=\frac{1}{2} u^{\mathrm{b}}(u) d t-p \in V^{*},
$$

and $\delta H / \delta \mu=u$. Using the Leibniz property of the Lie derivative, Stoke's theorem (with $\partial M=\emptyset)$ yields

$$
\begin{aligned}
& \left\langle b,-£_{u} D\right\rangle_{V}=-\int_{M} b £_{u}(\rho d V)=-\int_{M}\left[£_{u}(b \rho d V)-\left(£_{u} b\right) \rho d V\right] \\
& =-\int_{M} \mathbf{d i}_{u}(b \rho d V)+\int_{M}\left(\mathbf{i}_{u} \mathbf{d} b\right) \rho d V \\
& =\langle\mathbf{d} b \otimes D, u\rangle_{\mathfrak{X}}, \quad \forall D=\rho d V \in V, \quad \forall b \in V^{*}, \quad \forall u \in \mathfrak{X} .
\end{aligned}
$$

which implies that $b \diamond D=-\mathbf{d} b \otimes D \in \mathfrak{X}^{*}$. It follows that

$$
\mathbb{E}\left[\frac{\delta \ell}{\delta D}\right] \diamond D=\mathbb{E}\left[\frac{1}{2} \mathbf{d}\left(u^{b}(u)\right)-\mathbf{d} p\right] \otimes D .
$$

The LA SALT equations are obtained by substituting this relation into (2.3), which in this case read

$$
\mathrm{d}\left(u^{\mathrm{b}} \otimes D\right)+\mathfrak{f}_{\mathrm{d} X_{t}}\left(u^{\mathrm{b}} \otimes D\right)=\mathbb{E}\left[\frac{1}{2} \mathbf{d}\left(u^{\mathrm{b}}(u)\right)-\mathbf{d} p\right] \otimes D \mathrm{~d} t \quad \text { and } \mathrm{d} D+\mathfrak{d}_{\mathrm{d} X_{t}} D=0 .
$$

Recalling $D=\rho d V \in V$ and that the Lie derivative satisfies the Leibniz rule with respect to the tensor product, we have

$$
\mathrm{d} u^{b}+\mathfrak{d}_{\mathrm{d} X_{t}} u^{\mathrm{b}}=\mathbf{d}\left(\mathbb{E}\left[\frac{1}{2} u^{\mathrm{b}}(u)-p\right]\right) \mathrm{d} t \quad \text { and } \quad \mathrm{d} \rho+\mathfrak{d}_{\mathrm{d} X_{t}} \rho+\operatorname{div}\left(\mathrm{d} X_{t}\right) \rho=0 .
$$

We now discuss how the 'pressure' is determined. We understand 'incompressibility' in this example to mean that the density $D=\rho d V$, which is Lie-advected by $d X_{t}$, satisfies $\rho \equiv 1$. Since the density $\rho$ satisfies

$$
\mathrm{d} \rho+£_{\mathrm{d} X_{t}} \rho=-\operatorname{div}\left(\mathrm{d} X_{t}\right) \rho,
$$

the only term driving the density away from unity is

$$
\operatorname{div}\left(\mathrm{d} X_{t}\right)=\operatorname{div}\left(\mathbb{E}\left[u_{t}\right]\right) \mathrm{d} t+\operatorname{div}\left(\xi^{(k)}\right) \mathrm{d} W_{t}^{(k)}=\operatorname{div}\left(\mathbb{E}\left[u_{t}\right]\right) \mathrm{d} t,
$$


where we have used that $\operatorname{div}\left(\xi^{(k)}\right)=0$ by assumption. Therefore, to impose that $\rho \equiv 1$, we require $\operatorname{div}\left(\mathbb{E}\left[u_{t}\right]\right)=0$. Upon setting $\tilde{p}:=\frac{1}{2} u^{b}(u)-p$, we arrive at the LA SALT Euler equations on $M$

$$
\begin{aligned}
\mathrm{d} u^{\mathrm{b}}+\mathfrak{E}_{\mathbb{E}[u]} u^{\mathrm{b}} \mathrm{d} t+\sum_{k} \mathfrak{\xi}_{\xi^{(k)}} u^{\mathrm{b}} \circ \mathrm{d} W_{t}^{(k)} & =\mathbf{d} \mathbb{E}[\tilde{p}] \mathrm{d} t \\
\delta \mathbb{E}\left[u^{\mathrm{b}}\right] & =0,
\end{aligned}
$$

where $\delta: \Omega^{1} \rightarrow \Omega^{0}$ is the codifferential operator, and where $\tilde{p}$ is non-locally determined to enforce $\delta \mathbb{E}\left[u^{b}\right]=0$. Recalling that $\mathbf{P}$ is the divergence-free projection operator and letting $\mathbf{P}^{\mathbb{E}}=I-\mathbf{Q} \mathbb{E}$, we can then rewrite the equations as

$$
\mathrm{d} u^{\mathrm{b}}+\mathbf{P}^{\mathbb{E}} \mathfrak{E}_{\mathbb{E}[u]} u^{\mathrm{b}} \mathrm{d} t+\sum_{k} \mathbf{P}^{\mathbb{E}} \mathfrak{f}_{\xi(k)} u^{\mathrm{b}} \circ \mathrm{d} W_{t}^{(k)}=0
$$

We note that this equation is equivalent to (1.10) in the Introduction, where $u^{b}=u \cdot d x$. Thus, it clear that the pressure required to maintain incompressibility of $\mathbb{E}\left[u_{t}\right]$ is deterministic. The reason for treating the pressure to be $\mathbb{F}$-adapted a priori rather than deterministic is to maintain the connection with the structure introduced in the general class of LA SALT models (2.7) introduced in Sect. 2.1. However, given the dynamics, only the expectation of the pressure can be recovered, and hence it is only the expected pressure which plays a role. Thus, hereafter, we will simply denote $\pi_{t}=\mathbb{E}[p]$ and write

$$
\begin{aligned}
\mathrm{d} u^{\mathrm{b}}+\mathfrak{£}_{\mathbb{E}[u]} u^{\mathrm{b}} \mathrm{d} t+\sum_{k} £_{\xi^{(k)}} u^{\mathrm{b}} \circ \mathrm{d} W_{t}^{(k)} & =\mathbf{d} \pi \mathrm{d} t \\
\delta \mathbb{E}\left[u^{\mathrm{b}}\right] & =0 .
\end{aligned}
$$

Remark 3 Instead of defining incompressibility to mean that $\rho \equiv 1$, we could have defined it to mean that the stochastic velocity $u=\left(u^{b}\right)^{\sharp}=v$ is divergence free. While we do not pursue this here, it can easily be incorporated into our framework by defining $\mathfrak{X}=\mathfrak{X}_{\text {div }}$ and $\mathfrak{X}^{*}=\Omega^{1} / \mathbf{d} \Omega^{0}$ and letting

$$
\ell(u)=\int_{M} g(u, u) d V=\int_{M} u^{\mathrm{b}}(u) d V .
$$

In this case, the LA SALT Euler motion equation reads, in the notation of Appendix 2,

$$
\mathrm{d} u^{b}+\mathbf{P} \mathfrak{E}_{\mathbb{E}[u]} u^{\mathrm{b}} \mathrm{d} t+\sum_{k} \mathbf{P} £_{\xi(k)} u^{\mathrm{b}} \circ \mathrm{d} W_{t}^{(k)}=0,
$$

or, equivalently, upon substituting $\mathbf{P}=I-\mathbf{Q}$, we have

$$
\mathrm{d} u^{\mathrm{b}}+\mathfrak{f}_{\mathbb{E}[u]} u^{\mathrm{b}} \mathrm{d} t+\sum_{k} £_{\xi(k)} u^{\mathrm{b}} \circ \mathrm{d} W_{t}^{(k)}=\mathbf{d} p \mathrm{~d} t+\sum_{k} \mathbf{d} q^{(k)} \mathrm{d} W_{t}^{(k)} \quad \text { and } \delta u^{\mathrm{b}}=0,
$$

where

$$
\mathbf{d} p=\mathbf{Q} \mathfrak{E}_{\mathbb{E}[u]} u^{b}+\mathbf{Q} \sum_{k}\left(\mathfrak{£}_{\xi(k)}\right)^{2} u^{b} \text { and } \mathbf{d} q^{k}=\mathbf{Q} \mathfrak{\xi}_{\xi(k)} u^{b}
$$

We remark that the analytical results stated in Sect. 3.3 for the model (3.2) on $M=\mathbb{T}^{d}$ are also true for this model. 


\subsection{Vorticity Dynamics}

Let $\omega=d u^{b} \in \Omega^{2}$ denote the vorticity two-form. Applying the exterior derivative $\mathbf{d}$ to (3.2) and using the property that it commutes with the Lie derivative yields

$$
\mathrm{d} \omega+\mathfrak{d}_{\mathrm{d} X_{t}} \omega=\mathrm{d} \omega+\mathfrak{E}_{\mathbb{E}[u]} \omega \mathrm{d} t+\sum_{k} £_{\xi(k)} \omega \circ \mathrm{d} W_{t}^{(k)}=0 .
$$

In computing the fluctuations of $\omega$, we may treat $\omega$ as an advected variable $a \in \Omega^{2}$, and thus from (2.15), we find

$$
\frac{1}{2} \partial_{t} \mathbb{E}\left[\left|\omega^{\prime}\right|_{L^{2}}^{2}\right]-\left\langle\mathbb{E}\left[\star \omega^{\prime} \diamond \omega^{\prime}\right], \mathbb{E}[u]\right\rangle_{\mathfrak{X}}=-\frac{1}{2} \sum_{k}\left\langle\mathbb{E}\left[\star \omega^{\prime} \diamond\left(\mathfrak{f}_{\xi(k)} \omega^{\prime}\right)+\star \mathfrak{\xi}_{\xi(k)} \omega \diamond \omega\right], \xi^{(k)}\right\rangle_{\mathfrak{X}} .
$$

Therefore, the correlates $\xi^{(k)}$ play a decisive role in balancing the spatially integrated variances of the fluctuations of the vorticity.

Three Dimensions In $3 \mathrm{D}$, the vorticity $\omega$ may be identified with the vector $\vec{\omega}=\sharp \star \omega \in \mathfrak{X}$. Using the identity $\left[\sharp \star, £_{v}\right]=0$ (see, e.g., appendix section A.6. of [36]), we find that $\vec{\omega}$ is governed by the following system:

$\mathrm{d} \vec{\omega}+£_{\mathrm{d} X_{t}} \vec{\omega}=\mathrm{d} \vec{\omega}+\left[\mathrm{d} X_{t}, \vec{\omega}\right]=\mathrm{d} \vec{\omega}-\operatorname{ad}_{\mathrm{d} X_{t}} \vec{\omega}=\mathrm{d} \vec{\omega}-\operatorname{ad}_{\mathbb{E}[u]} \vec{\omega} \mathrm{d} t-\frac{1}{2} \sum_{k} \operatorname{ad}_{\xi(k)} \vec{\omega} \circ \mathrm{d} W_{t}^{(k)}=0$.

Following the analysis of Sect. 2.3 and using the definition (B.3), we find

$$
\begin{aligned}
& \frac{1}{2} \frac{\mathrm{d}}{\mathrm{d} t} \mathbb{E}\left[\left|\vec{\omega}^{\prime}\right|_{L^{2}}^{2}\right]+\left(\mathbb{E}\left[\operatorname{ad}_{\vec{\omega}_{t}^{\prime}}^{\dagger} \vec{\omega}_{t}^{\prime}\right], \mathbb{E}\left[u_{t}\right]\right)_{L^{2}} \\
& \quad+\frac{1}{2} \sum_{k}\left(\mathbb{E}\left[\operatorname{ad}_{\vec{\omega}_{t}^{\prime}}^{\dagger} \operatorname{ad}_{\xi(k)}^{\dagger} \vec{\omega}_{t}^{\prime}+\operatorname{ad}_{\vec{\omega}_{t}}^{\dagger} \operatorname{ad}_{\xi(k)} \vec{\omega}_{t}\right], \xi^{(k)}\right)_{L^{2}}=0 .
\end{aligned}
$$

Although the three-dimensional vorticity dynamics discussed so far is geometrically meaningful, it is not particularly illuminating for understanding the qualitative behaviour of the properties of its vorticity solutions. In two dimensions, we can obtain much more information about the vorticity solution behaviour.

Two Dimensions The vorticity in 2D may be transformed to a scalar using the Hodge-star operator $\omega=\star \omega \in \Omega^{0}$. (We abuse notation and still call it $\omega$ for the purposes of this section.) Again, using the identity $\left[\star, £_{v}\right]=0$ (see, e.g., appendix section A.6. of [36]) the 2D vorticity dynamics may be expressed as a scalar transport equation given by

$$
\mathrm{d} \omega+£_{d X_{t}} \omega=0,
$$

where we emphasize because $\omega \in \Omega^{0}$, in a local chart $(U, \phi)$ with coordinates $x=$ $\left(x^{1}, \ldots x^{d}\right)$ and $\left.v \in \mathfrak{X}\right|_{U}$, we have $£_{v} \omega_{t}=v^{i} \partial_{x^{i}} \omega_{t}$. Thus, we have the push-forward relation $\omega_{t}=\left(\phi_{t}\right)_{*} \omega_{0}$, where $\left\{\phi_{t}\right\}$ is the stochastic flow associated with $d X_{t}$. Moreover, for all $f \in \Lambda^{0}$,

$$
\mathrm{d} f(\omega)+£_{d X_{t}} f(\omega)=0,
$$

and $f\left(\omega_{t}\right)=\left(\phi_{t}\right)_{*} f\left(\omega_{0}\right)$. In addition, upon recalling that $\operatorname{div}_{g}\left(d X_{t}\right)=0$, we find

$$
\int_{M} f\left(\omega_{t}\right) d A=\int_{M}\left(\phi_{t}\right)_{*} f\left(\omega_{0}\right) d A=\int_{\phi_{t}(M)}\left(\phi_{t}\right)_{*} f\left(\omega_{0}\right) d A=\int_{M} f\left(\omega_{0}\right) \phi_{t}^{*} d A=\int_{M} f\left(\omega_{0}\right) d A,
$$


where, in this section, we $d A$ replaces $d V$ to denote area. In particular, choosing $\phi(x)=x^{p}$ we find that all of the $L^{p}$-norms of the solution are conserved.

We now want to investigate the fluctuations of the vorticity:

$$
\int_{M} \mathbb{E}\left[\left(\omega_{t}^{\prime}\right)^{2}\right] d A=\int_{M} \mathbb{E}\left[\omega_{t}^{2}\right] d A-\int_{M}\left(\mathbb{E}\left[\omega_{t}\right]\right)^{2} d A .
$$

We begin by computing the first term $\int_{M} \mathbb{E}\left[\omega_{t}^{2}\right] d V$. Upon taking $f(x)=x^{2}$ in (3.5), we find

$$
\int_{M} \omega_{t}^{2} d A=\int_{M} \omega_{0}^{2} d A \Rightarrow \int_{M} \mathbb{E}\left[\omega_{t}^{2}\right] d A=\int_{M} \mathbb{E}\left[\omega_{0}^{2}\right] d A
$$

Taking the expectation of (3.4) yields

$$
\partial_{t} \mathbb{E}[\omega]+\mathfrak{E}_{\mathbb{E}[u]} \mathbb{E}[\omega]=\frac{1}{2} \sum_{k} £_{\xi^{(k)}} £_{\xi(k)} \mathbb{E}[\omega] .
$$

A computation similar to (3.1) shows that for all $v \in \mathfrak{X}$ with $\operatorname{div}_{g} v=0$ and $f, g \in \Omega^{0}$,

$$
\left(£_{v} f, g\right)_{L^{2}\left(\Omega^{0}\right)}=-\left(f, £_{v} g\right)_{L^{2}\left(\Omega^{0}\right)}
$$

and hence $\left(\mathfrak{f}_{v} f, f\right)_{L^{2}\left(\Omega^{0}\right)}=0$. Thus, using the divergence-free property of $d X_{t}$ yields

$$
\int_{M}\left(\mathbb{E}\left[\omega_{t}\right]\right)^{2} d A=\int_{M}\left(\mathbb{E}\left[\omega_{0}\right]\right)^{2} d A-\sum_{k} \int_{M}\left(£_{\xi^{(k)}} \mathbb{E}\left[\omega_{t}\right]\right)^{2} d A,
$$

which implies that the magnitude $|\mathbb{E}[\omega]|$ of the expected vorticity will decay to zero in the absence of forcing, provided that the vector fields $\left\{\xi^{(k)}\right\}_{k \in \mathbb{N}}$ span $\mathfrak{X}$. Therefore, upon substituting (3.7) and (3.9) into (3.6), we find that fluctuations $\omega^{\prime}=\omega-\mathbb{E}[\omega]$ satisfy

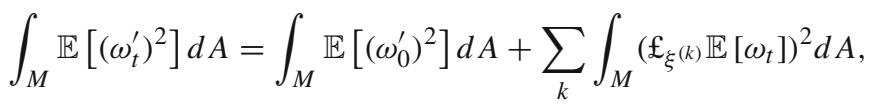

or equivalently

$$
\frac{\mathrm{d}}{\mathrm{d} t} \int_{M} \mathbb{E}\left[\left(\omega_{t}^{\prime}\right)^{2}\right] d A=\sum_{k} \int_{M}\left(\mathfrak{£}_{\xi^{(k)}} \mathbb{E}\left[\omega_{t}\right]\right)^{2} d A .
$$

This means that the conserved total enstrophy in (3.7) transforms from the mean into the fluctuations for 2D LA SALT vorticity dynamics. The same phenomenon occurs for the magnitude of the body angular momentum in the finite-dimensional example of rigid-body dynamics, see Sect. 4.1). On the other hand, the total enstrophy itself is preserved because it is a Casimir for the Lie-Poisson structure of the 2D LA SALT Euler equation, given by

$$
\mathrm{d} C(\omega)=-\int_{M} \omega J\left(\frac{\delta C}{\delta \omega}, \mathbb{E}\left[\frac{\delta H}{\delta \omega}\right] \mathrm{d} t+\sum_{k} \xi^{(k)} \circ \mathrm{d} W_{t}^{(k)}\right) d A=0,
$$

with Jacobian operator defined by $J(f, h) d A=\mathbf{d} f \wedge \mathbf{d} h$ just as it is for the deterministic Euler system.

Remark 4 (Synopsis) Total enstrophy is the spatial integral of the square of vorticity. It is preserved as a result of being a Casimir of the LA SALT system for the Lie-Poisson bracket expressed in (3.11), so its expectation is preserved by LA SALT. However, consider the enstrophy of the expected vorticity, i.e., the spatial integral of the square of the expectation of vorticity in (3.9). According to the results of this paragraph, the enstrophy of the expected 
vorticity decays exponentially in time, while the vorticity variance in (3.10) increases exponentially in time. Consequently, one would expect the probability distribution of enstrophy on a level set of enstrophy in the space of Casimir functionals tends to become more diffuse. This means that as time proceeds the probability density for the enstrophy will tend to a constant on the level set of the enstrophy corresponding to the initial condition. Thus, transport noise in 2D LA SALT vorticity dynamics causes a growth of uncertainty and a loss of information as the probability distribution for enstrophy delocalises and tends toward the analog for enstrophy of the microcanonical distribution in statistical physics.

In other words, one may regard the expected vorticity equations for 2D LA SALT in (3.8) as a dissipative system embedded into a larger conservative system (3.4). From this viewpoint, the interaction dynamics of the two components of the full LA SALT system dissipates the enstrophy of the mean vorticity by converting it into fluctuations, while preserving the mean total enstrophy. This dynamics results because the total (mean plus fluctuation) vorticity field is being linearly transported along the mean velocity in (3.4), while the mean vorticity field is decaying in $2 \mathrm{D}$ dissipative motion (3.8). This is the nature of stochastic coadjoint motion for the LA SALT Euler fluid vorticity equation in 2D, expressed in (3.11). Namely, the Casimirs are preserved by the full LA SALT dynamics, while the equations for the expected dynamics contain dissipative terms. Dissipation in the dynamics of the expected enstrophy is accompanied by loss of information (or increasing uncertainty) as the variance (expected fluctuation enstrophy) grows and the probability distribution of the enstrophy delocalises and tends toward a constant invariant measure.

\subsection{Helicity Preservation in 3D}

The LA SALT Euler fluid motion (3.2) for $u^{b} \in \Omega^{1}$ and vorticity equation (3.3) for $\omega=$ $\mathbf{d} u^{\mathrm{b}} \in \Omega^{2}$, together read

$$
\left(\mathrm{d}+\mathfrak{f}_{\mathrm{d} X_{t}}\right) u^{\mathrm{b}}=-\mathbf{d} \pi \text { and }\left(\mathrm{d}+\mathfrak{d}_{\mathrm{d} X_{t}}\right) \omega=0 .
$$

Using that the Lie-derivative satisfies the Leibniz rule with respect to the wedge product, that the exterior derivative $\mathbf{d}$ is an antiderivation, that $\mathbf{d}^{2}=0$, and the Stratonovich product rule, we obtain

$$
\left(\mathrm{d}+\mathfrak{f}_{\mathrm{d} X_{t}}\right)\left(u^{\mathrm{b}} \wedge \omega\right)=-\mathbf{d} p \wedge \omega=-\mathbf{d}(p \wedge \omega) .
$$

Then using the Kunita-Itô-Wentzell formula [12] and that $\partial M=\emptyset$, we find

$$
\begin{aligned}
\mathrm{d} \int_{M}\left(u^{\mathrm{b}} \wedge \omega\right) & =\mathrm{d} \int_{\phi_{t}(M)}\left(u^{\mathrm{b}} \wedge \omega\right)=\int_{M}\left(\mathrm{~d}+\mathfrak{f}_{\mathrm{d} X_{t}}\right)\left(u^{\mathrm{b}} \wedge \omega\right) \\
& =-\int_{M} \mathbf{d}(p \wedge \omega)=-\int_{\partial M}(p \wedge \omega)=0,
\end{aligned}
$$

where $\left\{\phi_{t}\right\}$ is the stochastic flow corresponding to $d X_{t}$. This integral quantity is known as the helicity. Its topological significance as the linkage number for lines of vorticity in a volume preserving fluid flow is discussed by Arnold in [37,38]. The preservation of helicity for SALT and LA SALT dynamics emphasizes once again the central role played by the Kelvin circulation integral in fluid dynamics. 


\subsection{Well-Posedness and Regularization by Non-locality in Probability Space}

In this section, we take $M$ to be the flat torus $\mathbb{T}^{d}=\mathbb{R}^{d} / \mathbb{Z}^{d}$. It is possible to generalize all these results to hold on compact smooth Riemannian manifolds $(M, g)$ without boundary. For the purpose of introducing notation, let $E$ denote an arbitrary Banach space. Let $L_{\omega}^{2} E$ denote the Lebesgue Bochner space $L^{2}(\Omega, \mathcal{F}, \mathbb{P} ; E)$. For a given time $T>0$ and $p \in[1, \infty]$, let $L_{T}^{p} E$ denote the Lebesgue Bochner space $L^{p}([0, T], \mathcal{B}([0, T]), \Omega ; E)$, where $\Omega$ is the Lebesgue measure on $\mathbb{R}$. For given $d \in\{2,3\}$ and $m \in \mathbb{N}$, let $H_{x}^{m}$ denote the Sobolev space of $m$-times weakly differentiable functions from $\mathbb{T}^{d}$ to $\mathbb{R}^{d}$ that have square integrable derivatives up to and including order $m$. We let $H_{\sigma}^{m} \subset H_{x}^{m}$ denote the subspace of weakly divergence-free functions and $\mathbf{P} \in \mathcal{L}\left(H_{x}^{m} ; H_{\sigma}^{m}\right)$ denote the bounded linear projection of $H_{x}^{m}$ onto $H_{\sigma}^{m}$ (i.e., the Leray or solenoidal projection). For a given time $T>0$, let $C_{T} E$ denote the space of strongly continuous functions from $[0, T]$ to $E$. For a given $m \in \mathbb{N}$, let $C_{x}^{m} E$ denote the space of $m$-times continuously differentiable functions from $\mathbb{T}^{d}$ to $E$. Let $C_{\sigma}^{m} E^{d} \subset C_{x}^{m} E^{d}$ denote subspace of divergence-free functions. We let $C_{x}^{\infty} E=\cap_{m} C_{x}^{m} E$ and similarly we define $C_{\sigma}^{\infty} E^{d}$. Let $\ell_{2}^{d}$ denote the space of square-summable sequences in $\mathbb{R}^{d}$. All of the above spaces introduced above are Banach spaces $V$, and we denote the corresponding norms by $|\cdot|_{V}$. Moreover, we let $(\cdot, \cdot)$ denote the $L^{2}\left(\mathbb{T}^{d} ; \mathbb{R}^{d}\right)$-inner product and $(\cdot, \cdot)_{H_{x}^{m}}=\left((I-\Delta)^{\frac{m}{2}} \cdot,(I-\Delta)^{\frac{m}{2}} \cdot\right)$ denote the inner product on $H_{x}^{m}$.

Fix a terminal time $T>0$ and $d \in\{2,3\}$. For a given divergence-free forcing term $f:[0, T] \times \mathbb{T}^{d} \rightarrow \mathbb{R}^{d}$, divergence-free initial condition $u_{0}: \Omega \times \mathbb{T}^{d} \rightarrow \mathbb{R}^{d}$, and family of divergence-free vector fields $\left\{\xi^{(k)}\right\}_{k \in \mathbb{N}}: \mathbb{T}^{d} \rightarrow \ell_{2}^{d}$, we consider the equation for an $\mathbb{F}$-adapted vector field $u: \Omega \times[0, T] \times \mathbb{T}^{d} \rightarrow \mathbb{R}^{d}$ and scalar field. pressure $p: \Omega \times[0, T] \times \mathbb{T}^{d} \rightarrow \mathbb{R}^{d}$ given by

$$
\left\{\begin{array}{l}
\mathrm{d} u_{t}+£_{\mathbb{E}\left[u_{t}\right]}^{T} u_{t} \mathrm{~d} t+\sum_{k} £_{\xi(k)}^{T} u_{t} \circ \mathrm{d} W_{t}^{(k)}=\left(-\nabla \pi_{t}+f_{t}\right) \mathrm{d} t, \\
\operatorname{div} \mathbb{E}\left[u_{t}\right]=0, \\
\left.u_{t}\right|_{t=0}=u_{0},
\end{array}\right.
$$

where $\pi_{t}=\mathbb{E}[p], £_{v}^{T} u=\operatorname{ad}_{v}^{\dagger} u=\left(\mathfrak{f}_{v} u^{\mathrm{b}}\right)^{\sharp}=\left(\operatorname{ad}_{v}^{*} u^{\mathrm{b}}\right)^{\sharp}$ is defined by

$$
£_{v}^{T} u_{t}:=v \cdot \nabla u_{t}+(\nabla v)^{T} \cdot u_{t},
$$

or, more explicitly, as $\left(\mathfrak{E}_{v}^{T} u_{t}\right)^{i}:=v^{j} \partial_{j} u^{i}+\left(\partial_{i} v^{j}\right) u^{j}$. We interpret (3.12) in the Itô-formulation:

$$
\mathrm{d} u_{t}+£_{\mathbb{E}\left[u_{t}\right]}^{T} u_{t} \mathrm{~d} t+\sum_{k} £_{\xi(k)}^{T} u_{t} \mathrm{~d} W_{t}^{(k)}=\left(\frac{1}{2} \sum_{k} £_{\xi(k)}^{T}\left(£_{\xi(k)}^{T} u_{t}\right)-\nabla \pi_{t}+f_{t}\right) \mathrm{d} t .
$$

A straightforward computation shows that for $\xi, v: \mathbb{T}^{d} \rightarrow \mathbb{R}^{d}$,

$$
\begin{aligned}
\mathfrak{f}_{\xi}^{T}\left(\mathfrak{f}_{\xi}^{T} v\right) & =(\xi \cdot \nabla) \xi \cdot \nabla v+(\xi \otimes \xi):(\nabla \otimes \nabla) v+2 \nabla \xi \cdot(\xi \cdot \nabla) v+\nabla((\xi \cdot \nabla) \xi) \cdot v \\
& =\partial_{i}\left(a^{i j} \partial_{j} u^{\alpha}\right)+b^{i \alpha j} \partial_{i} u^{j}+c^{\alpha \beta} u^{\beta},
\end{aligned}
$$

where

$$
a^{i j}:=\xi^{i} \xi^{j}, \quad b^{i \alpha j}=2 \xi^{i} \partial_{\alpha} \xi^{j}, \quad c^{\alpha \beta}:=\left(\partial_{\alpha} \xi^{i}\right) \partial_{i} \xi^{\beta}+\xi^{i} \partial_{i \alpha} \xi^{\beta}, \quad i, j, \alpha, \beta \in\{1, \ldots, d\},
$$

and repeated-indices are summed-over.

Taking the expectation of (3.13) yields a closed equation for the deterministic $v_{t}=\mathbb{E}\left[u_{t}\right]$ given by

$$
\partial_{t} v+\mathbf{P} £_{v}^{T} v=\mathbf{P} \frac{1}{2} \sum_{k} £_{\xi^{(k)}}^{T}\left(£_{\xi^{(k)}}^{T} v\right)+\mathbf{P} f_{t}
$$


Equation (3.15) for $\mathbb{E}\left[u_{t}\right]$ generalizes the classical $d$-dimensional Navier-Stokes equations which appear as a special case when $\xi^{(k)}:=\sqrt{2 v} e_{k}, k=1,2,3, \ldots, d$ and $\xi^{(k)}:=0$ otherwise. We term these equations (3.15) the Lie-Laplacian Navier-Stokes equations (LL $N S)$.

Assumption 1 There exist a $\kappa>0$ such that

$$
\kappa|y|^{2} \leq \frac{1}{2} \sum_{k} y^{i} \xi_{i}^{(k)}(x) \xi_{j}^{(k)}(x) y^{j}, \quad \forall x, y \in \mathbb{T}^{d} .
$$

Assumption $2(\mathrm{~m})$ For given $m \in \mathbb{N}, u_{0} \in H_{\sigma}^{m}, \xi \in C_{\sigma}^{m+2} \ell_{2}^{d}$, and $f \in L_{T}^{2} H_{\sigma}^{m-1}$.

Definition 1 (Solution of LA SALT Euler) We say that $u$ is a solution of (3.12) on the interval $\left[0, T^{*}\right]$ if $u$ is a weakly continuous $H_{x}^{1}$-valued $\mathbb{F}$-adapted process such that $u \in L_{\omega}^{2} L_{T^{*}}^{2} H_{x}^{1}$ and $\mathbb{E}[u] \in L_{T^{*}}^{2} H_{x}^{2} \cap L_{T^{*}}^{\infty} H_{x}^{1}$ and for all $\phi \in C_{x}^{\infty} \mathbb{R}^{d}, \mathbb{P}$-a.s. for all $t \in\left[0, T^{*}\right]$,

$$
\begin{aligned}
\left(u_{t}, \phi\right)= & \left(u_{0}, \phi\right)+\int_{0}^{t}\left[-\sum_{k}\left(£_{\xi(k)}^{T} u_{s}, £_{\xi(k)} \phi\right)+\left(-\mathfrak{£}_{\mathbb{E} u_{s}}^{T} u_{s}+f_{s}\right)-\left(\pi_{s}, \nabla \cdot \phi\right)\right] \mathrm{d} s \\
& -\sum_{k} \int_{0}^{t}\left(£_{\xi(k)}^{T} u_{s}, \phi\right) \mathrm{d} W_{s}^{(k)},
\end{aligned}
$$

where

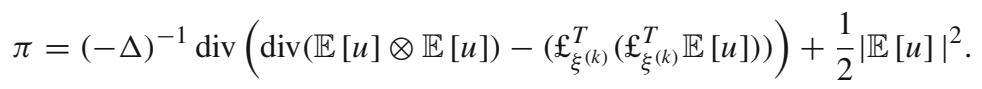

We remark that the assumption of $u \in L_{\omega}^{2} L_{T^{*}}^{2} H_{x}^{1}$ together with standard embeddings, Jensen's inequality and the convexity of norms give that $\mathbb{E}[u] \in L_{T^{*}}^{2} L_{x}^{4}$ in $d=2,3$. Therefore $\pi \in L_{T^{*}}^{1} L_{x}^{2}$ by elliptic regularity (see proof) and all above terms are well defined. Our analytical results are as follows.

Theorem 1 (Well-posedness of LA SALT Euler) Let $d \in\{2,3\}$ and Assumption 1 and 2 ( $m$ )

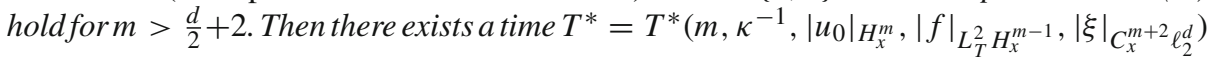
and a unique solution $u$ of LA SALT on $\left[0, T^{*}\right]$ satisfying $u \in L_{\omega}^{2} L_{T^{*}}^{\infty} H_{x}^{n-1}$ for $n:=$ $\lfloor m-d / 2\rfloor$. Furthermore, the solution $u$ is weakly continuous in time in $H_{x}^{n-1}$ and strongly continuous in $H_{x}^{n-2}$. If $d=2$, then $T^{*}=\infty$. Moreover, if $d=3$, there is a positive number

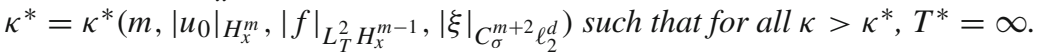

The proof of Theorem 1 proceeds as follows. We first solve (3.15) for $v=\mathbb{E}[u]$, and then solve the linear equation (3.13) for $u$. Accordingly, we need a solution theory for the deterministic LL-NS, which we state below. Note that, owing to (3.14) and (3.16), the Lie-Laplacian $\frac{1}{2} \sum_{k} £_{\xi^{(k)}}^{T}\left(£_{\xi^{(k)}}^{T} \cdot\right)$ is the sum of a divergence-form second-order uniformly elliptic operator $\partial_{i}\left(a^{i j} \partial_{j} \cdot\right)$ and a non-diagonal and non-symmetric first-order linear differential operator $b^{i} \partial_{i}+c$. Thus, from the standpoint of well-posedness and solution properties, the arguments to treat LL NS are completely analogous to those used to study the usual Navier-Stokes equation. The only additional ingredient is the solution estimate (3.18). For this reason, we only give a sketch of the proof of Theorem 2 and refer reader to the sources [39] and [40] for more details. 
Theorem 2 (Well-posedness of LL NS) Let $d \in\{2,3\}$ and Assumption 1 and $2(\mathrm{~m})$ hold for $m \geq 1$. Then there exists a time $T^{*}=T^{*}\left(m, \kappa^{-1},\left|u_{0}\right|_{H_{x}^{m}},|f|_{L_{T}^{2} H_{x}^{m-1}},|\xi|_{C_{x}^{m+2} \ell_{2}^{d}}\right)$ and a unique strong solution of (3.15) on $\left[0, T^{*}\right]$ satisfying $v \in C_{T^{*}} H_{\sigma}^{m} \cap L_{T^{*}}^{2} H_{\sigma}^{m+1}$. If $d=2$, then $T^{*}=\infty$. Moreover, if $d=3$, there is a positive number $\kappa^{*}=$

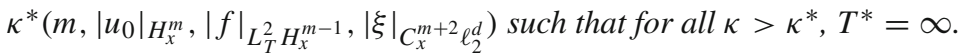

Remark 5 (Loss of regularity in Theorem 1) The loss of regularity the solution $u$ (i.e., $u$ is not necessarily in $H^{m}$ ) is an artifact of our method of proof. We apply a Sobolev solution theory for the linear stochastic transport theorem in which the coefficients in the drift of the SPDE are assumed to be at least $C^{1}$. We recall that $£_{\mathbb{E}[u]}^{T} u=\mathbb{E}[u] \cdot \nabla u+(\nabla \mathbb{E}[u])^{T} \cdot u$, and hence we need at least $\mathbb{E}[u] \in C_{x}^{2} \mathbb{R}^{d}$. It is plausible that one might be able to avoid the regularity loss by establishing a regularization by noise result for a linear stochastic transport system or by developing some other non-linear solution scheme.

Remark 6 (Large viscosity and global strong solutions) It is well-known that a global strong solution of the Navier-Stokes equation exists for sufficiently small initial data and forcing, or equivalently, sufficiently large viscosity. The lower bound on the viscosity coefficient can be made explicit in terms of the initial data and forcing, and we refer the reader to [39] or Chapter 5 of [40] for more details. As explained above, the double Lie-Laplacian is a uniformly elliptic operator with ellipticity constant $\kappa$ (see Assumption 1). Following the method of proof in the case of standard Laplacian dissipation, one can obtain a lower bound $\kappa^{*}$ for Lie-Laplacian dissipation depending on the initial data, forcing, and bounds on the coefficients $\xi$.

Remark 7 (Continuity of the solution maps) Standard techniques can be used to show that the solution map is continuous in initial data, forcing, and noise correlates both for the Lie-Laplacian Navier-Stokes equation

$$
\begin{aligned}
S_{L L N S}: H_{\sigma}^{m} \times L_{T}^{2} H_{\sigma}^{m-1} \times C_{\sigma}^{m+2} \ell_{2}^{d} & \rightarrow v \in C_{T^{*}} H_{\sigma}^{m} \cap L_{T^{*}}^{2} H_{\sigma}^{m+1} \\
\left(v_{0}, \xi, f\right) & \mapsto v,
\end{aligned}
$$

and, consequently, for the LA SALT Euler equations

$$
\begin{aligned}
S_{L A S A L T}: H_{\sigma}^{m} \times L_{T}^{2} H_{\sigma}^{m-1} \times C_{\sigma}^{m+2} \ell_{2}^{d} & \rightarrow v \in L_{\omega}^{2} C_{T^{*}} H_{x}^{n-1} \cap L_{\omega}^{2} L_{T^{*}}^{\infty} H_{x}^{n} \\
\left(u_{0}, \xi, f\right) & \mapsto u .
\end{aligned}
$$

Remark 8 If $\xi \in C_{\sigma}^{\infty} \ell_{2}^{d}, f \in C_{T}^{\infty} C_{\sigma}^{\infty}$, then $\left.\left.v \in C^{\infty}(] 0, T^{*}\right] \times \mathbb{T}^{d} ; \mathbb{R}^{d}\right)$ for any $\varepsilon>0$. See [39], Thm 7.5.

We now sketch the proofs of these two results.

Sketch of Proof of Theorem 2 It follows, for example, from Lemmas 3.6 and 3.7, and the argument on page 3779 of [41] (see, also, Lemma 5.1 in [42]) that there exist a constant $C=C\left(d, m, \kappa^{-1},|\xi|_{C_{x}^{m+2} \ell_{2}^{d}}\right)$ such that for all $u \in H_{x}^{m}$,

$$
\frac{1}{2} \sum_{k}\left(\left(£_{\xi^{(k)}}^{T}\right)^{2} u, u\right)_{H_{x}^{m}} \leq-\kappa^{\prime}|\nabla u|_{H_{x}^{m}}^{2}+C|u|_{H_{x}^{m}}^{2},
$$

for all $\kappa^{\prime}<\kappa$. The proof then follows from a simple modification of standard arguments, see e.g., Chapters 6 and 7 of [39] or Chapter 5 of [40]. Although we do not state them, the dependence of the local existence time and requisite large viscosity for global existence can all be made explicit in terms of $T$ and the size of data and forcing. 
Sketch of Proof of Theorem 1 Owing to Theorem 3.3 in [41] (see, also, Theorem 3.1 in [42]), if $u_{0} \in H_{x}^{n}, \mathbb{E}[u] \in L_{T^{*}}^{\infty} C_{x}^{n+1}, \xi \in C_{x}^{n+2} \ell_{2}^{d}$, and $f, \nabla \pi \in L_{T^{*}}^{2} H_{x}^{n}$, then there exists a solution $u$ of LA SALT on the interval $[0, T]^{*}$ such that $u \in L_{\omega}^{2} L_{T^{*}}^{2} H_{x}^{n}$. Moreover, $u$ is weakly continuous in $H_{x}^{n}$ and strongly continuous in $H_{x}^{n-1}$. These results are based on the following a priori estimate: there is a constant $C=C\left(d, m,|\mathbb{E}[u]|_{L_{T^{*}}^{\infty} C_{x}^{n+1}},|\xi|_{C_{x}^{n+1} \ell_{2}^{d}}\right)$ such that for all $u \in H^{n}$,

$$
\left(£_{\mathbb{E}[u]}^{T} u, u\right)_{H_{x}^{n}}+\frac{1}{2} \sum_{k}\left(\left(£_{\xi^{(k)}}^{T}\right)^{2} u, u\right)_{H_{x}^{n}}+\frac{1}{2} \sum_{k}\left|£_{\xi(k)}^{T} u\right|_{H_{x}^{n}}^{2} \leq C|u|_{H_{x}^{n}}^{2} .
$$

By virtue of the Sobolev embedding theorem and Theorem 2, if $u_{0} \in H_{\sigma}^{m}, f \in L_{T}^{2} H_{\sigma}^{m-1}$, and $\xi \in C_{\sigma}^{m+2} \ell_{2}^{d}$ for $m>d / 2+n+1$, then $\mathbb{E}[u] \in L_{T^{*}}^{\infty} C_{\sigma}^{n+1}$.

Taking the divergence of both sides of (3.13), we obtain the expression (3.17) for the expected pressure. Using standard estimates of elliptic PDE in Sobolev spaces (see, e.g., Thm III 4.1 and 4.2 in [40]), we find

$$
|\pi|_{H_{x}^{m-1}} \leq \frac{3}{2}|\mathbb{E}[u] \otimes \mathbb{E}[u]|_{H_{x}^{m-1}}+\left|\operatorname{div}\left(\left(\mathfrak{f}_{\xi^{(k)}}^{T}\left(\mathfrak{\xi}_{\xi(k)}^{T} \mathbb{E}[u]\right)\right)\right)\right|_{H_{x}^{m-3}} .
$$

Noting that $m-1>n+\frac{d}{2}$, by the Banach-algebra property of $H_{x}^{m-1}$ (see, e.g., Lemma 3.4 in [43]), there is a constant $C=C(d, m)$ such that $|\mathbb{E}[u] \otimes \mathbb{E}[u]|_{H_{x}^{m-1}} \leq C|\mathbb{E}[u]|_{H_{x}^{m-1}}^{2}$. Moreover, since $\operatorname{div} \mathbb{E}[u]=0$, it follows from Lemma 3.6 and the argument on page 3778 of [41] that there is a constant $C=C\left(d, m,|\xi|_{C_{x}^{m} \ell_{2}^{d}}\right)$ such that $\left|\operatorname{div}\left(\left(£_{\xi^{(k)}}^{T}\right)^{2} \mathbb{E}[u]\right)\right|_{H_{x}^{m-3}} \leq$ $C|\mathbb{E}[u]|_{H_{x}^{m-1}}$. Thus, we obtain

$$
\int_{0}^{T^{*}}\left|\nabla \pi_{t}\right|_{H_{x}^{n}}^{2} d t \leq \int_{0}^{T^{*}}\left|\pi_{t}\right|_{H_{x}^{m-1}}^{2} d t \leq C \int_{0}^{T^{*}}\left|\mathbb{E}\left[u_{t}\right]\right|_{H_{x}^{m-1}}^{2} d t<\infty,
$$

which gives $\nabla \pi \in L_{T^{*}}^{2} H_{x}^{n}$.

We now turn our attention to uniqueness. If $u_{1}, u_{2}$ are solutions of LA SALT, then $\mathbb{E}\left[u_{1}\right]$ and $\mathbb{E}\left[u_{2}\right]$ are strong solutions of LL-NS. Thus, $\mathbb{E}\left[u_{1}\right]=\mathbb{E}\left[u_{2}\right]$ since strong solutions are unique by Theorem 2. It then follows from the uniqueness of linear stochastic transport equations that $u_{1}=u_{2}$.

Remark 9 In fact, estimates of the form (3.19) hold for more general tensor fields. Lemma 3.7 along with the argument on page 3779 of [41] (see, also, Lemma 5.1 in [42]) directly imply that for all vector fields $v \in \mathfrak{X}$, there is constant $C=C\left(d, m,|v|_{C^{m+2}}\right)$ such that for all $\tau \in \mathcal{T}_{s}^{r}$, we have

$$
\left(£_{v}^{2} \tau, \tau\right)_{H^{m}\left(\mathcal{T}_{s}^{r}\right)}+\left|£_{v} \tau\right|_{H^{m}\left(\mathcal{T}_{s}^{r}\right)}^{2} \leq C|\tau|_{H^{m}\left(\mathcal{T}_{s}^{r}\right)}^{2}
$$

and for all $\alpha \in \Omega^{k}$,

$$
\left(£_{v}^{2} \alpha, \alpha\right)_{H^{m}\left(\Omega^{k}\right)}+\left|£_{v} \tau\right|_{H^{m}\left(\Omega^{k}\right)}^{2} \leq C|\tau|_{H^{m}\left(\Omega^{k}\right)}^{2} .
$$

Indeed, estimates of this form are derived in [41] and [42] for a first-order differential operator acting on functions $\phi: \mathbb{T}^{d} \rightarrow \mathbb{R}^{d^{\prime}}$ of the form

$$
(\mathcal{L} \phi)^{\alpha}=v^{j} \partial_{j} \phi^{\alpha}+\Omega^{\alpha j} \phi^{j},
$$

where $\xi \in C^{m+1}\left(\mathbb{T}^{d} ; \mathbb{R}^{d}\right)$ and $\Omega \in C^{m+1}\left(\mathbb{T}^{d} ; \mathbb{R}^{d^{\prime} \times d^{\prime}}\right)$. That is, there is constant $C=$ $C\left(d, m,|v|_{C^{m+1}}\right)$ such that (3.20) holds with $£_{v}$ replaced with $\mathcal{L}$ and $\tau$ or $\alpha$ replaced with 
$\phi$. One can see that the highest-order part (first-order) of $\mathcal{L}$ acts diagonally, which is why the Lie-derivative is a particular case. We remark also that estimates of this type were first derived for scalar functions in Lemma 2.1 of [44] (see, also, Lemma 4.3 in [45]). In fact, estimates given in Lemma 5.1 of [42] generalize such estimates to $L^{p}$-Sobolev spaces.

Remark 10 (Method of characteristics solution and representation) Let $\alpha>0$. If $b \in$ $L_{T^{*}}^{\infty} C_{x}^{2+\alpha}$ and $\xi \in C_{x}^{3+\alpha} \ell_{2}^{d}$, then there exists a stochastic flow of $C_{x}^{2+\alpha^{\prime}}$-diffeomorphisms $\phi=\left\{\phi_{s, t}\right\}$ for $\alpha^{\prime}<\alpha$ satisfying

$$
d \phi_{s, t}(x)=b\left(\phi_{s, t}(x)\right) \mathrm{d} t+\sum_{k} \xi^{(k)}\left(\phi_{s, t}(x)\right) \circ \mathrm{d} W_{t}^{(k)}, \quad \phi_{s, s}(x)=x \in \mathbb{T}^{d} .
$$

We take $b=\mathbb{E}[u]$, which is sufficiently regular provided Assumption 1 and 2 (m) hold for $m>\frac{d}{2}+2+\alpha$ by Theorem 2 . We denote the spatial inverse of the flow (i.e, the back-to-labels map) by $A_{t}=\phi_{0, t}^{-1}$. The inverse can be shown directly to satisfy (see, e.g., Theorem 2.4 in [46])

$$
\mathrm{d} A_{t}(x)=\mathbb{E}\left[u_{t}\right](x) \cdot \nabla A_{t}(x) \mathrm{d} t+\sum_{k} \xi^{(k)}(x) \cdot \nabla A_{t}(x) \circ \mathrm{d} W_{t}^{(k)}, \quad A_{0}(x)=x .
$$

It is easy to verify that $J_{t}^{-1}=\left(\nabla \phi_{0, t}\right)^{-1}$ satisfies

$\mathrm{d} J_{t}^{-1}(x)=-J_{t}^{-1}(x) \nabla \mathbb{E}\left[u_{t}\right]\left(\phi_{t}(x)\right) \mathrm{d} t-J_{t}^{-1}(x) \sum_{k} \nabla \xi^{(k)}\left(\phi_{t}(x)\right) \circ \mathrm{d} W_{t}^{(k)}, \quad J_{0}^{-1}(x)=I$.

Noting that $J_{t}^{-1}\left(A_{t}\right)=\nabla A_{t}$, we find (see, e.g., Theorem 2.2 in [47]))

$$
\begin{aligned}
& u_{t}(x)=\left(\nabla A_{t}(x)\right)^{T}\left[u_{0}\left(A_{t}(x)\right)+\Psi_{t}\left(A_{t}(x)\right)\right], \\
& \Psi_{t}(x)=\int_{0}^{t} J_{s}^{-1}(x)\left(f_{s}\left(\phi_{s}(x)\right)+\nabla \pi_{t}\left(\phi_{s}(x)\right)\right) \mathrm{d} s .
\end{aligned}
$$

In geometric notation, we may write the above as

$$
u_{t}^{\mathrm{b}}(x)=\left(\phi_{0, t}\right)_{*}\left(u_{0}^{\mathrm{b}}(x)+\psi^{\mathrm{b}}(x)\right),
$$

where $\psi^{b}$ is defined in terms of $f^{b}$ and $\mathbb{E}[d p]$, where $d p \in \Omega^{1}$ is exterior differential of $p$. This representation is closely related to the representation derived for the SALT system without a forcing term that is discussed in Remark 6 of [3]. In fact, in [3], the authors derive a stochastic representation for the solution $\mathbb{E}[u]$ of (3.15) akin to the Constantin-Iyer representation [2]:

$$
\mathbb{E}\left[u_{t}\right]=\mathbb{E}\left[\mathbf{P}\left[\left(\nabla A_{t}(x)\right)^{T} u_{0}\left(A_{t}(x)\right)\right]\right] .
$$

In fact, in those works, $u_{t}$ is identified as a stochastic Weber velocity and the representation (3.22) can directly be obtained from (3.21) for the 'Weber velocity' by taking expectation.

\section{Further Examples of LA SALT Systems}

This section illustrates the applicability of the LA SALT approach for a wide range of dynamical equations. Section 4.1 discusses a finite dimensional example at the foundations of geometric mechanics. This is the rigid body in three dimensions. This example is a great source of intuition and intuition for dynamics in geometric mechanics, and we expect it 
to be foundational in the exploration of applications of SALT and LA SALT, as well. See [48] for the general theory of SALT for Euler-Poincaré equations in finite dimensions. The corresponding general theory for LA SALT remains to be established in finite dimensions.

Section 4.2 treats the simplest LA SALT fluid equation. This is the Burgers equation, a fundamental 1D nonlinear equation which has been used to develop insight and intuition throughout fluid dynamics. Here we sketch an open problem for the LA SALT version of the Burgers equation. Namely, what is the signature of the noise correlates $\xi^{(k)}$ in the zero noise limit of LA SALT Burgers equation?

Section 4.3 discusses the LA SALT version of the Camassa-Holm $(\mathrm{CH})$ equation for singular nonlinear shallow water waves which are effectively finite dimensional. The LA SALT CH equation is the simplest example of the LA SALT formulation for soliton equations, yet it also summons fundamental questions about the regularity of its solutions.

Finally, to illustrate the breadth of applicability of the LA SALT formulation, we discussion its application for the multi-physics problem of incompressible, vertically stratified magnetohydrodynamics (MHD) in three dimensions in Sect. 4.4. The MHD example is meant to sketch yet another open problem and to show that the LA SALT formulation can accommodate a wide range of fluid applications. We expect the pursuit of these open problems in the applications of LA SALT to be fruitful and useful for fluid dynamics.

\subsection{LA SALT Rigid Body: A Finite Dimensional Example Without Advection}

In this example, we take $\mathfrak{X}=\mathfrak{s o}(3) \cong \mathbb{R}^{3}$ and $\mathfrak{X}^{*}=\mathfrak{s o}^{*}(3) \cong \mathbb{R}^{3}$ with the dot-product pairing (i.e., there is no density component $\mathfrak{X}^{*}$ ). Upon choosing $\ell(\boldsymbol{\Omega})=\frac{1}{2} \boldsymbol{\Omega}^{T} \cdot I \boldsymbol{\Omega}$, where $\mathrm{I}=\operatorname{diag}\left(\mathrm{I}_{1}, \mathrm{I}_{2}, \mathrm{I}_{3}\right)$ is the moment of inertia in the body frame, we find $\Pi:=\frac{\delta \ell}{\delta \boldsymbol{\Omega}}=I \boldsymbol{\Omega}$. As explained in [49], the SALT formulation of the stochastic rotation of a rigid body in $\mathbb{R}^{3}$ is governed by

$$
\mathrm{d} \boldsymbol{\Pi}+\left(\boldsymbol{\Omega} \mathrm{d} t+\sum_{k} \xi^{(k)} \circ \mathrm{d} W_{t}^{(k)}\right) \times \boldsymbol{\Pi}=0,
$$

where $\boldsymbol{\Pi}$ is interpreted as the angular momentum vector and $\boldsymbol{\Omega}=\mathrm{I}^{-1} \boldsymbol{\Pi}$ as the angular velocity vector. If we replace $\boldsymbol{\Omega}$ by the expected angular velocity $\mathbb{E}[\boldsymbol{\Omega}]$, we obtain the LA SALT body dynamics

$$
\mathrm{d} \Pi+\mathbb{E}[\boldsymbol{\Omega}] \times \Pi \mathrm{d} t+\sum_{k} \xi^{(k)} \times \Pi \circ \mathrm{d} W_{t}^{(k)}=0 .
$$

In either the SALT or LA SALT body dynamics, one has

$$
\frac{\mathrm{d}}{\mathrm{d} t}|\boldsymbol{\Pi}|^{2}=0
$$

which follows from (4.1) and the permutation vector identity $a \cdot b \times c=c \cdot a \times b$. Therefore, the probability distribution for the SALT and LA SALT rigid body lies on a level set of $|\Pi|^{2}$. Thus, these dynamics represent stochastic coadjoint motion on a level set of the Casimir function of the Lie-Poisson bracket.

In the Itô representation, this stochastic motion equation (4.1) becomes

$$
\mathrm{d} \boldsymbol{\Pi}+\left(\mathbb{E}[\boldsymbol{\Omega}] \mathrm{d} t+\sum_{k} \xi^{(k)} \mathrm{d} W_{t}^{(k)}\right) \times \boldsymbol{\Pi}=\frac{1}{2} \sum_{k} \xi^{(k)} \times\left(\xi^{(k)} \times \boldsymbol{\Pi}\right) \mathrm{d} t .
$$


Taking the expectation yields

$$
\frac{\mathrm{d}}{\mathrm{d} t} \mathbb{E}[\boldsymbol{\Pi}]+\mathbb{E}[\boldsymbol{\Omega}] \times \mathbb{E}[\boldsymbol{\Pi}]=\frac{1}{2} \sum_{k} \xi^{(k)} \times\left(\xi^{(k)} \times \mathbb{E}[\boldsymbol{\Pi}]\right) .
$$

Upon again using the permutation vector identity, we find

$$
\frac{\mathrm{d}}{\mathrm{d} t}|\mathbb{E}[\Pi]|^{2}=-\sum_{k}\left|\xi^{(k)} \times \mathbb{E}[\Pi]\right|^{2} \mathrm{~d} t
$$

Thus, the magnitude $|\mathbb{E}[\Pi]|$ of the expected body angular momentum vector $\mathbb{E}[\Pi]$ will decay to zero in the absence of forcing. This means that $\mathbb{E}[\boldsymbol{\Pi}]$ itself and $\mathbb{E}[\boldsymbol{\Omega}]$ will also decay to zero, provided that $\left\{\xi^{(k)}\right\}_{k \in \mathbb{N}}$ span $\mathbb{R}^{3}$. To calculate the fluctuation dynamics of $\Pi^{\prime}:=\Pi-\mathbb{E}[\Pi]$, we subtract equation (4.3) from equation (4.1) to find

$$
\mathrm{d} \boldsymbol{\Pi}^{\prime}+\mathbb{E}[\boldsymbol{\Omega}] \times \boldsymbol{\Pi}^{\prime} \mathrm{d} t+\sum_{k} \xi^{(k)} \times \boldsymbol{\Pi} \mathrm{d} W_{t}^{(k)}=\frac{1}{2} \sum_{k} \xi^{(k)} \times\left(\xi^{(k)} \times \boldsymbol{\Pi}^{\prime}\right) \mathrm{d} t .
$$

By the Itô product rule we have

$$
\frac{1}{2} \mathrm{~d}\left|\boldsymbol{\Pi}^{\prime}\right|^{2}=\boldsymbol{\Pi}^{\prime} \cdot \mathrm{d} \boldsymbol{\Pi}^{\prime}+\frac{1}{2} \mathrm{~d}\left\langle\boldsymbol{\Pi}^{\prime}, \boldsymbol{\Pi}^{\prime}\right\rangle_{t}=\boldsymbol{\Pi}^{\prime} \cdot \mathrm{d} \boldsymbol{\Pi}^{\prime}+\frac{1}{2} \sum_{k}\left|\xi^{(k)} \times \boldsymbol{\Pi}\right|^{2} \mathrm{~d} t .
$$

Thus, upon taking the dot product of $\boldsymbol{\Pi}^{\prime}$ with (4.4), applying the Itô cross-variance formula implies

$$
\begin{aligned}
\frac{1}{2} \mathrm{~d}\left|\boldsymbol{\Pi}^{\prime}\right|^{2}+\boldsymbol{\Pi}^{\prime} \cdot \sum_{k} \xi^{(k)} \times \boldsymbol{\Pi} \mathrm{d} W_{t}^{(k)} & =\frac{1}{2} \sum_{k}\left(\boldsymbol{\Pi}^{\prime} \cdot \xi^{(k)} \times\left(\xi^{(k)} \times \boldsymbol{\Pi}^{\prime}\right)+\left|\xi^{(k)} \times \boldsymbol{\Pi}\right|^{2}\right) \\
& =\frac{1}{2} \sum_{k}\left(-\left|\xi^{(k)} \times \boldsymbol{\Pi}^{\prime}\right|^{2}+\left|\xi^{(k)} \times \boldsymbol{\Pi}\right|^{2}\right) \\
& =\frac{1}{2} \sum_{k}\left(2\left(\xi^{(k)} \times \mathbb{E}[\boldsymbol{\Pi}]\right) \cdot\left(\xi^{(k)} \times \boldsymbol{\Pi}\right)-\left|\xi^{(k)} \times \mathbb{E}[\boldsymbol{\Pi}]\right|^{2}\right) .
\end{aligned}
$$

Taking the expectation then yields

$$
\frac{\mathrm{d}}{\mathrm{d} t} \mathbb{E}\left[\left|\boldsymbol{\Pi}^{\prime}\right|^{2}\right]=\sum_{k}\left|\xi^{(k)} \times \mathbb{E}[\Pi]\right|^{2}
$$

and hence the fluctuations grow as time increases.

In summary, level sets of $|\Pi|^{2}$ are preserved, by (4.2). That is, the invariant measure for the motion is supported on the angular momentum sphere. This is the same result as for the SALT rigid body, treated in [49]. On the other hand, for LA SALT the variances grow monotonically, so the distribution of angular momentum on the angular-momentum sphere tends to become more diffuse. Consequently, one expects the probability distribution for the magnitude of the angular momentum of LA SALT rigid body to tend toward a constant on a level set of $|\Pi|^{2}$. This was proved to happen as well for the SALT rigid body in [49]. 


\subsection{The LA SALT Burgers Equation}

Fix, for simplicity $M=\mathbb{S}^{1}$. Choosing $\ell(u)=\frac{1}{2} \int_{S^{1}}|u|^{2} d x$, the one dimensional LA SALT Burgers equation reads

$$
\mathrm{d} u+\mathbb{E}\left[u_{t}\right] \partial_{x} u \mathrm{~d} t+\sum_{k} \xi^{(k)} \partial_{x} u \circ \mathrm{d} W_{t}^{(k)}=0 .
$$

The SALT Burgers equations (without the expectation on the drift velocity) were studied in [50] and it was shown that shocks form almost surely. On the other hand, the solutions of the LA SALT Burgers equation stay regular. Indeed, the expectation $v_{t}=\mathbb{E}\left[u_{t}\right]$ satisfies

$$
\partial_{t} v+v \partial_{x} v=\sum_{k} \xi^{(k)} \partial_{x}\left(\xi^{(k)} \partial_{x} v\right)
$$

which is a viscous Burgers equation. Thus, if the $\xi^{(k)}$ are sufficiently smooth and nondegenerate (3.16), then the above equation gives rise to a global smooth solution $v_{t}$. The full field is then recovered by a linear transport equation, as in the LA SALT Euler case. Thus, Burgers equation provides a clear example of regularization by 'non-locality' in probability space. Note also from the transport structure one has the representation

$$
u_{t}(x)=u_{0}\left(A_{t}(x)\right), \quad v_{t}=\mathbb{E}\left[u_{0}\left(A_{t}(x)\right)\right],
$$

where $A_{t}$ is the back-to-labels map defined in Remark 10. At the level of the mean $v_{t}$, the above reasoning generalizes the stochastic method of characteristics (Feynman-Kac formula) for the usual Burgers equation [2]. This representation was used in the work [51] to study the limit of vanishing viscosity for viscous Burgers. There, it was shown the Lagrangian trajectories in the zero noise/viscosity limit become non-unique backward in time due to stochastic splitting that occurs at shock points. This phenomenon, known as spontaneous stochasticity, has many implications for understand high-Reynolds number, turbulent physics such as Richardson particle dispersion [52-55], anomalous dissipation [21,22,56,57], and time-irreversibility [58-60]. It would be interesting to study the effect of the $\xi^{(k)}$ functions on the zero-noise limit of LA-SALT Burgers, as well as their signature on the spontaneously stochastic probability measure on generalized trajectories of the entropy solutions.

\subsection{LA SALT Camassa-Holm (CH) Equation}

Fix, for simplicity $M=\mathbb{S}^{1}$. Upon choosing $\ell(u)=\frac{1}{2} \int_{\mathbb{S}^{1}}\left(u^{2}+\alpha^{2}\left(\partial_{x} u\right)^{2}\right) d x$ and defining the Lie derivative of a one-form density $m=m(d x)^{2}$ by a vector field $\xi$ in 1D as

$$
£_{\xi} m=\left(\partial_{x} m+m \partial_{x}\right) \xi
$$

one finds the LA SALT CH equation

$$
\begin{aligned}
\mathrm{d} m & =-\left(\partial_{x} m+m \partial_{x}\right)\left(\mathbb{E}\left[\frac{\delta H}{\delta m}\right] d t+\sum_{k} \xi^{(k)} \circ \mathrm{d} W_{t}^{(k)}\right) \\
& =-\mathfrak{f}_{K * \mathbb{E}[m]} m \mathrm{~d} t-\sum_{k} £_{\xi^{(k)}} m \circ \mathrm{d} W_{t}^{(k)} .
\end{aligned}
$$


where $K(x)=\frac{1}{2} \exp (-|x| / \alpha)$ is the Green's function for the 1D Helmholtz operator $1-\alpha^{2} \partial_{x}^{2}$ and where the stochastic $\mathrm{CH}$ Hamiltonian is given by

$$
d h(m)=H(m) \mathrm{d} t+\sum_{k} \int_{\mathbb{S}^{1}} m \xi^{(k)} \circ \mathrm{d} W_{t}^{(k)} d x,
$$

with deterministic part

$$
H(m)=\frac{1}{2} \int_{\mathbb{S}^{1}} m K * m d x=\frac{1}{2} \int_{\mathbb{S}^{1}} \int_{\mathbb{S}^{1}} m(x) K\left(\left|x-x^{\prime}\right|\right) m\left(x^{\prime}\right) d x^{\prime} d x .
$$

The expected velocity $\mathbb{E}[u]=\mathbb{E}[\delta H / \delta m]$ is given in terms of expected momentum $\mathbb{E}[m]$ by $\mathbb{E}[u]=K * \mathbb{E}[m]$, with $m=u-\alpha^{2} u_{x x}$ and $u=K * m$.

As an Itô stochastic transport equation, (4.5) reads

$$
\mathrm{d} m=-£_{K * \mathbb{E}[m]} m \mathrm{~d} t-\sum_{k} £_{\xi^{(k)}} m \mathrm{~d} W_{t}^{(k)}+\frac{1}{2} \sum_{k} £_{\xi(k)}\left(£_{\xi(k)} m\right) \mathrm{d} t,
$$

and its expectation immediately yields the dissipative equation

$$
\partial_{t} \mathbb{E}[m]=-£_{K * \mathbb{E}[m]} \mathbb{E}[m]+\frac{1}{2} \sum_{k} £_{\xi^{(k)}}\left(£_{\xi^{(k)}} \mathbb{E}[m]\right) .
$$

The subsequent calculations for LA SALT CH would follow the path established in Sect. 2 for deriving the dynamics of the fluctuations (2.11) and the spatially integrated variance (2.16) for the LA SALT CH equation. Rather then follow that path here, though, we shall consider the reduction to a finite dimensional system of SDEs which are nonlocal in probability space, arising from the singular momentum map afforded by the Lie-Poisson structure for the LA SALT equation in (4.5) [61].

LA SALT CH Peakons The Stratonovich version of the LA SALT CH equation (4.5) admits singular solutions for the 1D momentum $m_{t}$. In particular, these singular solutions can be distributions of momentum on points in the real line. In previous work for the SALT version of the $\mathrm{CH}$ equation, the singular solutions (peakons) were found to form with positive probability [62]. The singular peakon solution Ansatz is given by [63]:

$$
m_{t}(x)=\sum_{a=1}^{N} p_{a}(t) \delta\left(x-q_{a}(t)\right) \text { and } u_{t}(x)=\sum_{a=1}^{N} p_{a}(t) K\left(x-q_{a}(t)\right),
$$

with $K(x)=\frac{1}{2} \exp (-|x| / \alpha)$. Substitution of the peakon solution Ansatz into the LA SALT $\mathrm{CH}$ equation (4.5) yields the following closed SDEs for the time-dependent parameters $q_{a}(t)$ and $p_{a}(t)$,

$$
\begin{aligned}
& \mathrm{d} q_{a}=\mathbb{E}\left[\left.u_{t}(x)\right|_{x=q_{a}}\right] d t+\sum_{k} \xi^{(k)}\left(q_{a}\right) \circ \mathrm{d} W_{t}^{(k)}, \\
& \mathrm{d} p_{a}=-p_{a} \mathbb{E}\left[\left.\frac{\partial u_{t}(x)}{\partial x}\right|_{x=q_{a}}\right] d t-\left.p_{a} \sum_{k} \frac{\partial \xi^{(k)}(x)}{\partial x}\right|_{\substack{x=q_{a} \\
\circ}} \mathrm{d} W_{t}^{(k)},
\end{aligned}
$$

with $u_{t}(x)$ given in (4.6).

Remark 11 Although peakons have been shown to emerge in the initial value problem for SALT CH with positive probability [48], the issue of whether peakons emerge for the LA 
SALT CH dynamics in (4.5) from confined initial conditions for velocity $u(x, 0)$ remains an open question at this time. However, if the initial condition contains only peakons, then it's clear from equation (4.7) that they persist, so long as the solution exists for their dynamics governed by the closed system of SDEs in (4.7) for any finite number of peakons. These interesting, but unfamiliar LA SALT CH peakon SDEs have yet to be studied. ${ }^{2}$

\subsection{Incompressible, Vertically Stratified 3D LA SALT Magnetohydrodynamics (MHD)}

Fix, for simplicity $M=\mathbb{T}^{3}$. To formulate a comprehensive example, we consider the LA SALT MHD equations for an incompressible stratified medium moving in three dimensions under constant acceleration of gravity, $g$. The corresponding equations for 3D LA SALT MHD are given by,

$$
\begin{array}{r}
\mathrm{d} u+\left(\mathrm{d} X_{t} \cdot \nabla\right) u+u_{j} \nabla \mathrm{d} X_{t}^{j}=-\nabla\left(\mathbb{E}[p]-\frac{1}{2} \mathbb{E}\left[|u|^{2}\right]\right) \mathrm{d} t-g \mathbb{E}[b] \hat{z} \mathrm{~d} t \\
+g z \nabla(b-\mathbb{E}[b]) \mathrm{d} t+\mathbb{E}[J] \times B \mathrm{~d} t \\
\mathrm{~d} b+\mathrm{d} X_{t} \cdot \nabla b=0, \quad \mathrm{~d} B-\operatorname{curl}\left(\mathrm{d} X_{t} \times B\right)+\mathrm{d} X_{t}(\operatorname{div} B)=0 .
\end{array}
$$

In these equations, the stratification is measured by buoyancy, $b$. The magnetic field $B$ is divergence free, so that $\operatorname{div} B=0$. The current density is given in terms of the magnetic field by $J:=\operatorname{curl} B$. Finally, the transport velocity is given by the LA SALT Stratonovich stochastic vector field,

$$
\mathrm{d} X_{t}=\mathbb{E}[u](x) \mathrm{d} t+\sum_{k} \xi^{(k)}(x) \circ \mathrm{d} W_{t}^{(k)} .
$$

We note that the constraint $\operatorname{div} B=0$ is preserved, if it holds initially, which we will assume henceforth. Also, it's clear that writing the corresponding equations in Itô form would be straightforward, given the amount of previous description above. The physical variables for 3D incompressible MHD are: momentum $\mu \in \Omega^{1} \otimes$ Den, mass density $D \in$ Den, buoyancy $b \in \Omega^{0}$ and magnetic flux $B \in \Omega^{2}$, with components,

$$
\mu=\mu \cdot d x \otimes d^{3} x, \quad D=\rho d^{3} x, \quad b=b, \quad \text { and } \quad B=B \cdot d S .
$$

That is, $V=\operatorname{Den} \otimes \Omega^{0} \otimes \Omega^{2}$ and $a=(D, b, B) \in V$. The Hamiltonian for deterministic $3 \mathrm{D}$ incompressible, vertically stratified MHD in terms of the physical variables is

$$
H(\mu, D, b, B)=\int_{\mathbb{T}^{3}}\left(\frac{1}{2 \rho}|\mu|^{2}+g \rho b z+\frac{1}{2}|B|^{2}+p(\rho-1)\right) d^{3} x,
$$

whose variational derivatives are given by

$$
\delta H(\mu, D, b, B)=\int_{\mathbb{T}^{3}}\left(\frac{\mu}{\rho} \cdot \delta \mu+\left(p-\frac{|\mu|^{2}}{2 \rho^{2}}+g b z\right) \delta \rho+g \rho z \delta b+B \cdot \delta B\right) d^{3} x,
$$

so that one finds $u=\mu / \rho$.

2 See [64] for numerical simulations of these equations. These simulations show the emergence of trains of peakons whose amplitudes (speeds) tend to decay in time. 
The entries in the Hamiltonian operator in equation (4.10) for 3D incompressible vertically stratified MHD are given in the first column by

$$
\begin{aligned}
£_{v}\left(\rho d^{3} x\right) & =\operatorname{div}(\rho v) d^{3} x, \quad £_{v}\left(\mu_{i} d x^{i} \otimes d^{3} x\right)=\left(\left(\partial_{j} \mu_{i}+\mu_{i} \partial_{j}\right) v^{j}\right) d x^{i} \otimes d^{3} x \\
£_{v} b & =v \cdot \nabla b, \quad £_{v}(B \cdot d S)=(-\operatorname{curl}(v \times B)+v(\operatorname{div} B)) \cdot d S .
\end{aligned}
$$

Then, by the definition of the diamond operator $\diamond: V^{*} \times V \rightarrow \mathfrak{X}^{*}$ in terms of the Lie derivative in (B.5), the remaining entries in the first row of the Hamiltonian operator involving diamond $(\diamond)$ are given by

$$
\frac{\delta H}{\delta D} \diamond D=D \nabla \frac{\delta H}{\delta D}, \quad \frac{\delta H}{\delta b} \diamond b=-\frac{\delta H}{\delta b} \nabla b, \quad \frac{\delta H}{\delta B} \diamond B=B \times \operatorname{curl} \frac{\delta H}{\delta B}-\frac{\delta H}{\delta B} \operatorname{div}(B) .
$$

These relations are sufficient to develop the dynamical equations (4.8) of the LA SALT 3D incompressible MHD example in Hamiltonian matrix form, as

$$
\mathrm{d}\left[\begin{array}{c}
\mu \\
D \\
b \\
B
\end{array}\right]=-\left[\begin{array}{cccc}
\mathfrak{f}_{(\cdot)} \mu(\cdot) \diamond D(\cdot) \diamond b(\cdot) \diamond B \\
\mathfrak{f}_{(\cdot)} D & 0 & 0 & 0 \\
\mathfrak{f}_{(\cdot)} b & 0 & 0 & 0 \\
\mathfrak{f}_{(\cdot)} B & 0 & 0 & 0
\end{array}\right]\left[\begin{array}{c}
\mathbb{E}[\delta H / \delta \mu] \mathrm{d} t+\sum_{k} \xi^{(k)} \circ \mathrm{d} W_{t}^{(k)} \\
\mathbb{E}[\delta H / \delta D] \mathrm{d} t \\
\mathbb{E}[\delta H / \delta b] \mathrm{d} t \\
\mathbb{E}[\delta H / \delta B] \mathrm{d} t
\end{array}\right] .
$$

The Casimirs for 3D vertically stratified incompressible MHD are

$$
C[b, B]=\int_{\mathbb{T}^{d}} \rho \Phi\left(b, \rho^{-1} B \cdot \nabla b\right) d^{3} x,
$$

for any differentiable function $\Phi \in \Lambda^{0}$.

Equations (4.9)-(4.10) deliver the system (4.8) for 3D incompressible, stratified LA SALT MHD in the framework established in section 2 for obtaining the complete dynamics of the expected solutions in equation (2.10), the fluctuations in equation (2.11) and the variances in equation (2.16). The semidirect-product Lie-Poisson Hamiltonian operator in equation (4.10) is identical to the corresponding operator for the deterministic case investigated in [7].

\section{Discussion}

In this paper, we propose a new class of stochastic field theories that are non-local in probability space and preserve certain analogues of the geometric properties of their deterministic counterparts. Core amongst the geometric properties is a stochastic version of Kelvin's circulation theorem. In these models, the momentum is Lie transported by the average of the associated velocity field plus a stochastic vector field. For this reason, we call our class of models LA SALT (Lagrangian averaged stochastic advection by Lie transport) theories.

We present a framework that encompasses a broad class of systems and derive the equations for the mean and fluctuation fields. Moreover, we describe in-depth the theory for an incompressible ideal fluid, LA SALT Euler. This theory has the special feature that the expected velocity field completely decouples dynamically, and the fluctuations are passively transported by the average solution. As a consequence, we show that the non-local (in probability space) nature of the transport velocity provides a mechanism for dissipation of the 
mean field and leads to a quantitative regularizing effect on the solutions. Finally, we offer further concrete examples of LA SALT systems and discuss the interest and possible lines of investigation into these models.

Let us now conclude with some remarks concerning potential future directions. First, it would be interesting to investigate whether the LA SALT models may be used to describe the coarse-scale features of a fluid exhibiting motions over a wide range of scales by (stochastically) parameterizing the effects of the unresolved small scales on the coarse features of the flow. For example, following the SALT approach, the vector fields $\xi^{(k)}(x)$ could potentially be determined from a data analysis or a calibration procedure involving direct numerical simulations of the equations of motion $[11,13,14]$. Another exciting direction would be to extend the LA SALT framework to allow $\xi^{(k)}$ to depend functionally on the large-scale field. In analogy to eddy viscosity-type models in the large eddy simulation (LES) community, a successful choice of such dependence may lead to a more robust model. Finally, it would be of great importance to offer a systematic derivation of the LA SALT as an effective theory either in a regime of wide-scale separation, where homogenization techniques may be employed as in $[1,18]$ ), or a truly multi-scale regime, where the rational may be related to a certain Markovian assumption within the Mori-Zwanzig formalism (see, e.g., [65] for a recent review).

Acknowledgements We are grateful for stimulating and encouraging discussions of the topics in this paper with C. J. Cotter, D. Crisan, A. Bethencourt de Leon, T. Nilssen and S. Takao. We particularly thank S. Takao for helpful discussions on the fluctuation variance dynamics in $\S 2.3$. Research of TD is partially supported by NSF-DMS Grant 1703997. DDH and JML are grateful for partial support by the EPSRC Standard Grant EP/N023781/1.

Open Access This article is licensed under a Creative Commons Attribution 4.0 International License, which permits use, sharing, adaptation, distribution and reproduction in any medium or format, as long as you give appropriate credit to the original author(s) and the source, provide a link to the Creative Commons licence, and indicate if changes were made. The images or other third party material in this article are included in the article's Creative Commons licence, unless indicated otherwise in a credit line to the material. If material is not included in the article's Creative Commons licence and your intended use is not permitted by statutory regulation or exceeds the permitted use, you will need to obtain permission directly from the copyright holder. To view a copy of this licence, visit http://creativecommons.org/licenses/by/4.0/.

\section{Appendices}

\section{A: Historical Background of the Geometric Approach to Fluid Mechanics}

In two papers published in 1966, Arnold changed the way we think about fluid dynamics, forever. In the papers $[66,67]$, Arnold showed that the solutions of the Euler fluid equations in a domain $M$ in fixed space, $M \subset \mathbb{R}^{n}$, can be mapped by the classic Lagrange-Euler representation to a time-dependent path on the manifold of volume-preserving diffeomorphisms acting on $\mathbb{R}^{n}$ (SDiff $\left(\mathbb{R}^{n}\right)$ ) which is geodesic with respect to the right-invariant metric on its tangent space given by the kinetic energy of the fluid. The kinetic energy metric is right-invariant because it is the $L^{2}$ norm of the right-invariant Eulerian velocity $u_{t}$ defined by

$$
\dot{g}_{t} x_{0}=u_{t}\left(g_{t} x_{0}\right)
$$

in which subscript $t$ denotes explicit time dependence. This $g_{t}$ is simply the Lagrangian flow map at time $t$ associated to the velocity field $u$. The Lagrangian fluid parcel trajectory is given by 


$$
x_{t}:=g_{t} x_{0} \in M \text { with } g_{0} x_{0}=x_{0} .
$$

Upon writing $u_{t}=\dot{g}_{t} g_{t}^{-1}$ one sees that the invariance of the Eulerian velocity corresponds to relabelling the Lagrangian parcel label $x_{0} \rightarrow y_{0}=h x_{0}$ for a fixed map $h \in \operatorname{SDiff}\left(\mathbb{R}^{n}\right)$. Thus, under any fixed map $h$ acting from the right we have $\dot{g}_{t} h\left(g_{t} h\right)^{-1}=\dot{g}_{t} g_{t}^{-1}$. Arnold's identification of the Euler fluid solutions as geodesics also brings in Hamilton's variational principle, in which right-invariance summons Noether's theorem for Lie group invariant variational principles.

Arnold's idea that Euler fluid flows could be lifted to time-dependent paths on $\operatorname{SDiff}\left(\mathbb{R}^{n}\right)$ has been continually fruitful. Already in 1970, Ebin and Marsden [68] used this idea to prove the local in time existence and uniqueness of the Euler fluid flows in $\mathbb{R}^{3}$. This is still the definitive analytical result for the Euler fluid equations. By 1985, Marsden and his collaborators had used the same idea to obtain the Lie-Poisson Hamiltonian formulation for ideal fluids with advected quantities and had recognised the role of its semidirect-product structure in establishing nonlinear stability for a wide class of fluid and plasma equilibria for continuum flows with advected quantities and additional physical fields [17,69]. Again, this development of nonlinear stability conditions followed Arnold's lead in [66] for the nonlinear stability of Euler fluid equilibria as critical points of a constrained variational principle for time dependent paths on the manifold $\operatorname{SDiff}\left(\mathbb{R}^{n}\right)$. For further explanation of these developments in the context of momentum maps, see [70,71].

Following an observation reported in 1901 by Poincaré, [72], Holm, Marsden and Ratiu [7] transferred the idea of symplectic reduction for Hamiltonian systems into the theory of reduction by Lie symmetries of the Lagrangian in Hamilton's principle and applied the resulting Euler-Poincaré equations to derive the general dynamics of fluid flows with advected quantities. This result again followed Arnold's lead in regarding fluid flows as curves on $\operatorname{SDiff}\left(\mathbb{R}^{3}\right)$, although the dynamics discussed in [7] takes place on $\operatorname{Diff}\left(\mathbb{R}^{3}\right)$ in the compressible case when volume is not preserved. An interesting feature in this particular development involves the Kelvin-Noether theorem. This theorem revealed that the momentum map on the Lagrangian side for the action corresponding to the relabelling symmetry was, in fact, the circulation integral in Kelvin's theorem for Euler's equations. Thus, the conservation of the Kelvin circulation integral for the Euler fluid equations was found to arise via Noether theorem from the symmetry of the Eulerian velocity in (A.1) under relabelling of the Lagrangian fluid parcels.

Now, the Lagrangian fluid parcels carry advected physical variables such as mass, heat, other thermodynamic quantities, buoyancy for stratification of fluid motion under gravity, magnetic field lines for MHD, etc. The introduction of spatially varying initial conditions for these advected quantities breaks the symmetry of the Euler kinetic energy Lagrangian under the full set of Lie group transformations by $G=\operatorname{Diff}\left(\mathbb{R}^{n}\right)$. In particular, the invariance of the Lagrangian is restricted to the "isotropy subgroups" $G_{a_{0}}:=\operatorname{Diff}_{a_{0}}\left(\mathbb{R}^{n}\right)$ of the full $\operatorname{Diff}\left(\mathbb{R}^{n}\right)$ Lie transformations. The isotropy subgroups $G_{a_{0}}$ are those which leave invariant the initial conditions $a_{0}$ chosen for the advected quantities. The advected quantities evolve by push-forward $a_{t}=a_{0} g_{t}^{-1}$ by the action of the entire $\operatorname{Diff}\left(\mathbb{R}^{n}\right)$. (Push-forward is pull-back by right action of $g_{t}^{-1}$.) Thus, symmetry breaking from $\operatorname{Diff}\left(\mathbb{R}^{n}\right)$ to $\operatorname{Diff} a_{0}\left(\mathbb{R}^{n}\right)$ leads to the identification of advected quantities as order parameters $a_{t} \in G / G_{a_{0}}$, where $G / G_{a_{0}}$ is the corresponding coset space of the broken symmetry under the action of $G$ by the remaining symmetry $G_{a_{0}}$ for $a_{0}$. This symmetry breaking of $\operatorname{Diff}\left(\mathbb{R}^{n}\right)$ for the Euler fluid equations implies that the Euler-Poincaré equations for flows with advected quantities acquire force terms of geometric origin, thereby leading to the so-called "diamond $(\diamond)$ terms" which will be discussed further below. Including these force terms arising from symmetry breaking 
implies that fluid solutions with advected quantities are no longer geodesic paths on Diff or SDiff. For an application of these ideas to complex fluids such as liquid crystals, see [73,74].

This history of the development of Arnold's SDiff flow concept and its extension to include advected fluid quantities provides the context of flows on Diff or SDiff for the introduction of the material addressed in the present paper. Because of its close connection to Lie symmetry transformations via Noether's theorem, this material can be addressed operationally and quite transparently from the viewpoint of Kelvin's theorem, by using pull-back by the time dependent flow. This operational interpretation arises naturally because of the physical connection of Kelvin's theorem to Newton's force law. Namely, the Kelvin circulation integral is the Noether quantity describing momentum distributed on closed material loops [7].

\section{B: Geometric Setting}

In this section, we define the elements of the geometric/probabilistic framework which facilitate the interpretation of our equations.

Basic Setting and Notation Let $(M, g)$ be a $C^{\infty}$, compact, oriented, $d$-dimensional Riemannian manifold with empty boundary $\partial M=\emptyset$. We will denote by $d V$ the Riemannian volume form on $M$ associated with the metric.

Let $\mathfrak{X}$ denote the space of smooth vector fields on $M, \mathcal{T}_{s}^{r}$ denote the space of smooth $r$-contravariant $s$-covariant tensor fields on $M$ with the tensor product denoted by $\otimes$. Let $\Omega^{k}$ denote the space of smooth exterior $k$-forms on $M$ with the wedge product denoted $\wedge$. Let d : $\Omega^{k} \rightarrow \Omega^{k+1}$ denote the exterior differential operator and $\mathbf{i}_{u}: \Omega^{k+1} \rightarrow \Omega^{k}$ the interior product for a given $u \in \mathfrak{X}$, both of which are antiderivations.

For a given vector field $u \in \mathfrak{X}$ and $\tau \in \mathcal{T}_{s}^{r}$, let $£_{X} \tau$ denote the Lie derivative of $\tau$ along $X$, which can be defined by $£_{X} \tau=\left.\frac{d}{d t}\right|_{t=0} \phi_{t}^{*} \tau$, where for each $t, \phi_{t}^{*} \tau$ denotes the pull-back of the tensor field $\tau$ by the flow map $\phi_{t}$ associated with $u$.

For a given tensor field $\alpha \in \Omega^{k}$ and $u \in \mathfrak{X}$, the Lie derivative satisfies Cartan's formula

$$
£_{u} \alpha=\mathbf{d}\left(\mathbf{i}_{u} \alpha\right)+\mathbf{i}_{u} \mathbf{d} \alpha .
$$

Moreover, in a local chart $(U, \phi)$ of $M$ with coordinates $\left(x^{1}, \ldots, x^{d}\right)$, one has

$$
£_{u} v=-\operatorname{ad}_{u} v=[u, v]=\left(u^{j} \partial_{x^{j}} v^{i}-v^{j} \partial_{x^{j}} u^{i}\right) \partial_{x^{i}}, \quad \text { for } u=u^{i} \partial x^{i}, v=\left.v^{i} \partial_{x^{i}} \in \mathfrak{X}\right|_{U},
$$

as well as

$$
\begin{aligned}
\left(\mathfrak{f}_{u} \omega\right)_{i_{1} \cdots i_{k}} & =u^{j} \partial_{x^{j}} \omega_{i_{1} \cdots i_{k}}+\omega_{j \cdots i_{k}} \partial_{x^{i_{1}}} u^{j}+\cdots+\omega_{i_{1} \cdots j} \partial_{x^{i_{k}}} u^{j}, \\
\text { for } \omega & =\left.\omega_{i_{1} \cdots i_{k}} d x^{i_{1}} \wedge \cdots \wedge d x^{i_{k}} \in \Omega^{k}\right|_{U}, i_{1}<\cdots<i_{k},
\end{aligned}
$$

and

$$
\begin{aligned}
& \left(\mathfrak{f}_{u} \tau\right)_{i_{1} \cdots i_{S}}^{j_{1} \cdots j_{r}}=u^{j} \partial_{x^{j}} \tau_{i_{1} \cdots i_{S}}^{j_{1} \cdots j_{r}}+\tau_{j \cdots i_{S}}^{j_{1} \cdots j_{s}} \partial_{x^{i_{1}}} u^{j}+\cdots+\tau_{i_{1} \cdots j}^{j_{1} \cdots j_{r}} \partial_{x^{i_{S}}} u^{j}+\tau_{i_{1} \cdots i_{S}}^{j_{1} \cdots j_{r}} \partial_{x^{j_{1}}} u^{j}+\cdots+\tau_{i_{1} \cdots i_{k}}^{j_{1} \cdots j_{x}} \partial_{x} u_{r} u^{j}, \\
& \text { for } \tau=\left.\tau_{i_{1} \cdots i_{s}}^{j_{1} \cdots j_{r}} \partial_{x^{1}} \otimes \cdots \otimes \partial_{x^{r}} \otimes d x^{i_{1}} \otimes \cdots \otimes d x^{i_{s}} \in \mathcal{T}_{s}^{r}\right|_{U} .
\end{aligned}
$$

Musical Isomorphisms, the Hodge Star Operator, and the Hodge Decomposition The metric $g$ induces an isomorphism between $\mathfrak{X}$ and $\Omega^{1}$; we denote by $b: \mathfrak{X} \rightarrow \Omega^{1}$ the index lowering map and by $\sharp: \Omega^{1} \rightarrow \mathfrak{X}$ the index raising map. Similarly, the indices of a tensor field or differential form can be raised or lowered by using the metric. Furthermore, the metric $g$ automatically induces Riemannian structures on the bundles $\mathcal{T}_{s}^{r}$ and $\Omega^{k}$, which we 
continue to denote by $g$. Indeed, for $\alpha, \beta \in \Omega^{1}, g(\alpha, \beta)=\alpha\left(\beta^{\sharp}\right)=\beta\left(\alpha^{\sharp}\right)=g\left(\alpha^{\sharp}, \beta^{\sharp}\right)$, and then the metric on $\mathcal{T}_{s}^{r}$ is defined by the tensor product $\otimes^{r} g \otimes \otimes^{s} g$.

The metric and orientation uniquely determines the Hodge star operator $\star: \Omega^{k} \rightarrow \Omega^{d-k}$, which satisfies $\alpha \wedge \star \beta=g(\alpha, \beta) \mu$ for all $\alpha, \beta \in \Omega^{k}$. The corresponding co-differential $\delta: \Omega^{k} \rightarrow \Omega^{k-1}$ is defined by $\delta=(-1)^{d(k+1)+1} \star \mathbf{d} \star$. The metric also induces a pre-inner product on $\mathcal{T}_{s}^{r}$ (and hence on $\Omega^{k}$ ) defined by

$$
\left(\tau, \tau^{\prime}\right)_{L^{2}\left(\mathcal{T}_{s}^{r}\right)}=\int_{M} g\left(\tau, \tau^{\prime}\right) d V
$$

for $\tau, \tau^{\prime} \in \mathcal{T}_{s}^{r}$. It can be shown that for all $\alpha, \beta \in \Omega^{k}$,

$$
(\alpha, \beta)_{L^{2}\left(\Omega^{k}\right)}=\int_{M} g(\alpha, \beta) d V=\int_{M} g(\alpha, \beta) \star 1=\int_{M} \alpha \wedge \star \beta,
$$

and that the co-differential $\delta$ is formally the adjoint of the differential $\mathbf{d}$ on $\cup_{k=0}^{d} \Omega^{k}$ with respect to this inner product.

Letting $L^{2}\left(\Omega^{k}\right)$ denote the space of Borel measurable square-integrable equivalent classes of functions $\Omega^{k}$ and equipping this space the inner product $(\cdot, \cdot)_{L^{2}\left(\Omega^{k}\right)}$ produces a Hilbert space. The Hodge decomposition (see e.g., Ch. 3 of [75]) associated with the Hodge Laplacian $\Delta=\mathbf{d} \delta+\delta \mathbf{d}$, then reads

$$
L^{2}\left(\Omega^{k}\right)=\overline{\delta \Omega^{k+1}} \oplus \overline{\mathbf{d} \Omega^{k-1}} \oplus \mathcal{H}^{k},
$$

where $\overline{(\cdot)}$ denotes the closure in $L^{2}\left(\Omega^{k}\right)$ of the corresponding set and $\mathcal{H}_{k}=\left\{\alpha \in \Omega^{k}\right.$ : $\Delta \alpha=0\}$ is a space of harmonic- $k$ forms, which is orthogonal to the other two components. Noting the identity $\mathbf{i}_{u} \mu=\star u^{b}$ for $u \in \mathfrak{X}$, we find that $\operatorname{div}_{g}(u)=-\delta u^{b}$, where $\operatorname{div}_{g}(u)$ is defined to be the unique element of $\Omega^{0}=C^{\infty}(M)$ such that

$$
£_{u} d V=\operatorname{div}_{g}(u) d V=\mathbf{d}\left(i_{u} d V\right) .
$$

Therefore, the Hodge decomposition of $L^{2}\left(\Omega^{1}\right)$ says that for every $u \in \mathfrak{X}$,

$$
u^{b}=\mathbf{P} u^{b}+\mathbf{Q} u^{b}+\mathbf{P}_{\mathcal{H}} u^{b},
$$

where the sharp of $\mathbf{P} u^{b}$ is a weakly divergence-free vector field, the sharp of $\mathbf{Q} u^{b}=\mathbf{d} p$ is the weak-gradient of a scalar function and the sharp of $\mathbf{P}_{\mathcal{H}} u^{b}$ is the harmonic-form part. It can also be shown that spaces $W^{m, p}\left(\Omega^{k}\right)$ or $C^{\alpha}\left(\Omega^{k}\right)$ of Sobolev or Hölder differential forms, respectively, admit Hodge decompositions in which there respective divergence-free or gradient-components have the same corresponding regularity (see, e.g., [76,77]).

Momentum, One-Form Densities, Weak Duals, Ad-star and Ad-dagger. The LA SALT theories presented in the text are based on Euler-Poincare (EPDiff) theory [7], in which the (weak) dual spaces of $\mathfrak{X}, \Omega^{k}, \mathcal{T}_{s}^{r}$, and $\mathcal{T}_{s}^{r} \otimes \Omega^{d}$ with respect to $L^{2}$-pairings play a prominent role. This is because the theory is founded on variational principles in which Gateux derivatives of functionals (e.g., the Lagrangian and constraints) involve $L^{2}$-norms (see (2.1)).

Since we have fixed a metric $g$, a natural candidate for the weak duals of $\mathfrak{X}, \Omega^{k}, \mathcal{T}_{s}^{r}$, and $\mathcal{T}_{s}^{r} \otimes \Omega^{d}$, respectively, can be defined using the $L^{2}$-pairing (B.1) to be $\mathfrak{X}, \Omega^{k}, \mathcal{T}_{s}^{r}$, and $\mathcal{T}_{s}^{r} \otimes \Omega^{d}$, respectively. That is, these spaces are weakly dual to themselves using the $L^{2}$-inner-product (based on the metric). However, the geometric structure and conservation laws such as Kelvin's theorem and its generalization are more readily apparent if one defines weak "geometric duals" as defined below and writes the Euler-Poincaré equations in terms of these geometric dual spaces. It is important to emphasize that the theory does not depend 
on how the duals are defined since one can always transform the equations among the various definitions.

Let Den $=\left\{v \in \Omega^{d}: v=\rho d V, \rho \in \Omega^{0}\right\} \subset \mathcal{T}_{d+1}^{0}$. We identify the weak (geometric) dual of $\mathfrak{X}$ with $\mathfrak{X}^{*}=\Omega^{1} \otimes$ Den via the pairing $\langle\cdot, \cdot\rangle_{\mathfrak{X}}: \mathfrak{X}^{*} \times \mathfrak{X} \rightarrow \mathbb{R}$ defined by

$$
\langle\mu, u\rangle_{\mathfrak{X}}:=\int_{M} \alpha(u) \rho d V, \quad \mu=\alpha \otimes \rho d V \in \mathfrak{X}^{*}, \quad u \in \mathfrak{X} .
$$

It follows that the formal adjoint of $\operatorname{ad}_{v}: \mathfrak{X} \rightarrow \mathfrak{X}$ relative to the pairing $\langle\cdot, \cdot\rangle_{\mathfrak{X}}$ is $\operatorname{ad}_{v}^{*}=\mathfrak{f}_{v}$; that is, for all $\mu \in \mathfrak{X}^{*}$ and $u, v \in \mathfrak{X}$,

$$
\left\langle\mu, \operatorname{ad}_{v} u\right\rangle_{\mathfrak{X}}=\left\langle\mathfrak{f}_{v} \mu, u\right\rangle_{\mathfrak{X}} .
$$

Indeed, owing to the definition of the Lie-derivative, the change of variable formula, and the natural properties of the tensor product and contraction, for all $\alpha \otimes \rho d V \in \mathfrak{X}^{*}$ and $u, v \in \mathfrak{X}$, we have

$$
\begin{aligned}
\left\langle\alpha \otimes \rho d V, \operatorname{ad}_{v} u\right\rangle_{\mathfrak{X}} & =\int_{M} \alpha\left(\operatorname{ad}_{v} u\right) \rho d V=-\left.\frac{d}{d \epsilon}\right|_{\epsilon=0} \int_{M} \alpha\left(\phi_{\epsilon}^{*} u\right) \rho d V \\
& =-\left.\frac{d}{d \epsilon}\right|_{\epsilon=0} \int_{M}\left(\left(\phi_{\epsilon}\right)_{*} \alpha\right)(u)\left(\phi_{\epsilon}\right)_{*}(\rho d V) \\
& =-\int_{M}\left[\left(£_{-v} \alpha\right)(u) \rho d V+\alpha(u) £_{-v}(\rho d V)\right] \\
& =\left\langle £_{v}(\alpha \otimes \rho d V), u\right\rangle_{\mathfrak{X}},
\end{aligned}
$$

in which $\left\{\phi_{\epsilon}\right\}$ is the flow generated by $v$. Moreover, for all $\mu \in \mathfrak{X}^{*}$ and $u, v \in \mathfrak{X}$, we have

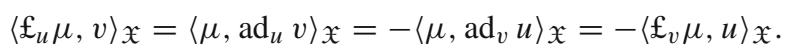

In geometric fluid dynamics, the momentum $\mu=\delta \ell / \delta u$ belongs to $\mathfrak{X}^{*}$.

Adjoint Operations The formal adjoint of $\operatorname{ad}_{v}: \mathfrak{X} \rightarrow \mathfrak{X}$ with respect to the $L^{2}(\mathfrak{X})$-innerproduct is denoted by $\mathrm{ad}_{v}^{\dagger}$. That is,

$$
\left(\operatorname{ad}_{v} u, w\right)_{L^{2}(\mathfrak{X})}=\left(u, \operatorname{ad}_{v}^{\dagger} w\right)_{L^{2}(\mathfrak{X})}, \quad \forall u, v, w \in \mathfrak{X} .
$$

It follows that for all $u, v, w \in \mathfrak{X}$,

$$
\left(\operatorname{ad}_{v}^{\dagger} u, w\right)_{L^{2}(\mathfrak{X})}=\left\langle\mathfrak{£}_{v}\left(u^{\mathrm{b}} \otimes d V\right), w\right\rangle_{\mathfrak{X}}=\left\langle\operatorname{ad}_{v}^{*}\left(u^{\mathrm{b}} \otimes d V\right), w\right\rangle_{\mathfrak{X}},
$$

and hence for all $\alpha \in \Omega^{1}$,

$$
\operatorname{ad}_{v}^{\dagger} u=\left(£_{v} u^{b}\right)^{\sharp}+\left(\operatorname{div}_{g} v\right) u, \quad \operatorname{ad}_{v}^{\dagger} \alpha^{\sharp}=\left(£_{v} \alpha\right)^{\sharp}+\left(\operatorname{div}_{g} v\right) \alpha^{\sharp} .
$$

Moreover, for all $u, v, w \in \mathfrak{X}$, we have

$$
\left(\operatorname{ad}_{v}^{\dagger} u, w\right)_{L^{2}(\mathfrak{X})}=\left(u, \operatorname{ad}_{v} w\right)_{L^{2}(\mathfrak{X})}=-\left(u, \operatorname{ad}_{w} v\right)_{L^{2}(\mathfrak{X})}=-\left(\operatorname{ad}_{w}^{\dagger} u, v\right)_{L^{2}(\mathfrak{X})} .
$$

For a given $\mu \in \mathfrak{X}^{*}$ and $D=\rho d V \in$ Den, we define the one-form $\frac{\mu}{D} \in \Omega^{1}$ by expressing the one-form density $\mu$ in terms of the volume-form $d V$ as

$$
\mu=\frac{\mu}{D} \otimes D=\frac{\mu}{D} \otimes \rho d V .
$$


Advected Variables and the Diamond Operation Let us define the weak (geometric) dual of $\Omega^{k}$ to be $\Omega^{d-k}$ via the pairing

$$
\langle\beta, \alpha\rangle_{\Omega^{k}}=\int_{M} \alpha \wedge \beta, \alpha \in \Omega^{k}, \beta \in \Omega^{d-k} .
$$

Let $V=\operatorname{Den} \oplus \oplus_{i=1}^{N} \Omega^{k_{i}}$ for $k_{i} \in\{0, \ldots, d\}$, which is the space in which the advected quantities will live. We denote by $V^{*}$ the (geometric) weak dual of $V$ relative to the weak non-degenerate pairing $\langle\cdot, \cdot\rangle_{V}: V^{*} \times V \rightarrow \mathbb{R}$ defined by

$$
\langle\cdot, \cdot\rangle_{V}=\langle\cdot, \cdot\rangle_{\Omega^{d}}+\sum_{i=1}^{N}\langle\cdot, \cdot\rangle_{\Omega^{k_{i}}}
$$

It follows that for all $a=\left\{a^{(i)}\right\}_{i=0}^{N}, b=\left\{b^{(i)}\right\}_{i=0}^{N} \in V$,

$$
(a, b)_{L^{2}(V)}:=\sum_{i=0}^{N}\left(a^{(i)}, b^{(i)}\right)_{L^{2}\left(\Omega^{\left.k_{i}\right)}\right.}^{2}=\sum_{i=0}^{N} \int_{M} a^{(i)} \wedge \star b^{(i)}=\langle\star b, a\rangle_{V}=\langle\star a, b\rangle_{V} .
$$

One can define geometric duals for tensor or tensor densities using tensor contraction and allow advected variables in $V$ to lie in these spaces as well. However, for simplicity, we will only treat the case of $k$-forms.

Let $\diamond: V^{*} \times V \rightarrow \mathfrak{X}^{*}$ be the bi-linear operation defined by the relation

$$
\langle b \diamond a, u\rangle_{\mathfrak{X}}=\left\langle b,-\mathfrak{£}_{u} a\right\rangle_{V}=\sum_{i=0}^{N}\left\langle b_{i},-\mathfrak{£}_{u} a^{(i)}\right\rangle, \quad \forall a=\left\{a^{(i)}\right\}_{i=0}^{N} \in V, b \in V^{*}, \forall u \in \mathfrak{X} .
$$

The diamond can be calculated on a case-by-case basis using the definition of the Liederivative (and Cartan's formula), Stoke's theorem, and the antiderivation properties of the exterior differential and insertion operators. See $\S 3$ and $\S 4$ for concrete examples including LA SALT Euler and MHD.

We define $\hat{\diamond}: V^{*} \times V \rightarrow \mathfrak{X}$ by $b \hat{\diamond} a=\left(\frac{1}{d V}(b \diamond a)\right)^{\sharp}$ for all $a \in V, b \in V^{*}$. It follows that for all $a \in V, b \in V^{*}$ and $u \in \mathfrak{X}$,

$$
(b \hat{\diamond} a, u)_{L^{2}(\mathfrak{X})}=\int_{M}\left(\frac{1}{d V}(b \hat{\diamond} a)\right)(u) d V=\langle b \diamond a, u\rangle_{\mathfrak{X}}=\left\langle b,-\mathfrak{f}_{u} a\right\rangle_{V} .
$$

\section{References}

1. Holm, D.D.: Variational principles for stochastic fluid dynamics. Proc. R. Soc. A 471(2176), 20140963, 19 (2015)

2. Constantin, P., Iyer, G.: A stochastic Lagrangian representation of the three-dimensional incompressible Navier-Stokes equations. Commun. Pure Appl. Math. 61(3), 330-345 (2008)

3. Drivas, T.D., Holm, D.D.: Circulation and energy theorem preserving stochastic fluids. Proc. R. Soc. Edinb. 1-39 (2019)

4. Mikulevicius, R., Rozovskii, B.L.: Stochastic Navier-Stokes equations for turbulent flows. SIAM J. Math. Anal. 35(5), 1250-1310 (2004)

5. Flandoli, F.: The interaction between noise and transport mechanisms in PDEs. Milan J. Math. 79(2), 543-560 (2011)

6. McKean Jr., H.P.: A class of Markov processes associated with nonlinear parabolic equations. Proc. Natl. Acad. Sci. USA. 56, 1907-1911 (1966)

7. Holm, D.D., Marsden, J.E., Ratiu, T.S.: The Euler-Poincaré equations and semidirect products with applications to continuum theories. Adv. Math. 137(1), 1-81 (1998) 
8. Foias, C., Holm, D.D., Titi, E.S.: The Navier-Stokes-alpha model of fluid turbulence. Physica D 152/153, 505-519 (2001). Advances in nonlinear mathematics and science

9. Foias, C., Holm, D.D., Titi, E.S.: The three dimensional viscous Camassa-Holm equations, and their relation to the Navier-Stokes equations and turbulence theory. J. Dyn. Differ. Equ. 14(1), 1-35 (2002)

10. Cotter, C.J., Gottwald, G.A., Holm, D.D.: Stochastic partial differential fluid equations as a diffusive limit of deterministic Lagrangian multi-time dynamics. Proc. R. Soc. A 473(2205), 20170388, 10 (2017)

11. Cotter, C., Crisan, D., Holm, D.D., Pan, W., Shevchenko, I.: Modelling uncertainty using circulation-preserving stochastic transport noise in a 2-layer quasi-geostrophic model. arXiv preprint arXiv:1802.05711 (2018)

12. de Leon, A.B., Holm, D., Luesink, E., Takao, S.: Implications of Kunita-Itô-Wentzell formula for $k$-forms in stochastic fluid dynamics. arXiv preprint arXiv:1903.07201 (2019)

13. Cotter, C., Crisan, D., Holm, D.D., Pan, W., Shevchenko, I.: Numerically modeling stochastic Lie transport in fluid dynamics. Multiscale Model. Simul. 17(1), 192-232 (2019)

14. Cotter, C., Crisan, D., Holm, D.D., Pan, W., Shevchenko, I.: A particle filter for stochastic advection by Lie transport (SALT): A case study for the damped and forced incompressible 2D Euler equation. arXiv preprint arXiv:1907.11884 (2019)

15. Alonso-Orán, D., de León, A.B., Holm, D., Takao, S.: Modelling the climate and weather of a 2D Lagrangian-averaged Euler-Boussinesq equation with transport noise. J. Stat. Phys. (to appear)

16. Hochgerner, S.: A Hamiltonian mean field system for the Navier-Stokes equation. Proc. R. Soc. A 474(2218), 20180178 (2018)

17. Holm, D.D., Kupershmidt, B.A.: Poisson brackets and Clebsch representations for magnetohydrodynamics, multifluid plasmas, and elasticity. Physica D 6(3), 347-363 (1983)

18. Gay-Balmaz, F., Holm, D.D.: Stochastic geometric models with non-stationary spatial correlations in Lagrangian fluid flows. J. Nonlinear Sci. 28(3), 873-904 (2018)

19. Crisan, D., Flandoli, F., Holm, D.D.: Solution properties of a 3D stochastic Euler fluid equation. J. Nonlinear Sci. 29(3), 813-870 (2019)

20. Jabin, P.-E., Wang, Z.: Mean Field Limit for Stochastic Particle Systems, pp. 379-402. Springer, Berlin (2017)

21. Drivas, T.D., Eyink, G.L.: A Lagrangian fluctuation-dissipation relation for scalar turbulence. Part I. Flows with no bounding walls. J. Fluid Mech. 829, 153-189 (2017)

22. Drivas, T.D., Eyink, G.L.: A Lagrangian fluctuation-dissipation relation for scalar turbulence. Part II. Wall-bounded flows. J. Fluid Mech. 829, 236-279 (2017)

23. Chen, S., Foias, C., Holm, D.D., Olson, E., Titi, E.S., Wynne, S.: Camassa-Holm equations as a closure model for turbulent channel and pipe flow. Phys. Rev. Lett. 81(24), 5338 (1998)

24. Chen, S., Foias, C., Holm, D.D., Olson, E., Titi, E.S., Wynne, S.: A connection between the CamassaHolm equations and turbulent flows in channels and pipes. Phys. Fluids 11(8), 2343-2353 (1999)

25. Chen, S., Foias, C., Holm, D.D., Olson, E., Titi, E.S., Wynne, S.: The Camassa-Holm equations and turbulence. Physica D 133(1-4), 49-65 (1999)

26. Revuz, D., Yor, M.: Continuous martingales and Brownian motion, vol. 293. Springer, Berlin (2013)

27. Prévôt, C., Röckner, M.: A concise course on stochastic partial differential equations, vol. 1905. Springer, Berlin (2007)

28. Elliott, C.M., Hairer, M., Scott, M.R.: Stochastic partial differential equations on evolving surfaces and evolving riemannian manifolds. arXiv preprint arXiv:1208.5958 (2012)

29. Gyöngy, I.: Stochastic partial differential equations on manifolds, I. Potential Anal. 2(2), 101-113 (1993)

30. Gyöngy, I.: Stochastic partial differential equations on manifolds II. nonlinear filtering. Potential Anal. 6(1), 39-56 (1997)

31. Krylov, N.V.: Itô's formula for the $L_{p}$-norm of stochastic $W_{p}^{1}$-valued processes. Probab. Theory Relat. Fields 147(3-4), 583-605 (2010)

32. Pardoux, É.: Sur des équations aux dérivées partielles stochastiques monotones. C. R. Acad. Sci. Paris Sér. A-B 275, A101-A103 (1972)

33. Pardoux, E.: Équations aux dérivées partielles stochastiques de type monotone. $\mathrm{PhD}$ thesis, Collège de France (1975)

34. Krylov, N.V., Rozovskiǔ, B.L.: Stochastic evolution equations. In: Current problems in mathematics, Vol. 14 (Russian), pp. 71-147, 256. Akad. Nauk SSSR, Vsesoyuz. Inst. Nauchn. i Tekhn. Informatsii, Moscow (1979)

35. Krylov, N.V.: A relatively short proof of Itô's formula for SPDEs and its applications. Stoch. Partial Differ. Equ. Anal. Comput. 1(1), 152-174 (2013)

36. Besse, N., Frisch, U.: Geometric formulation of the Cauchy invariants for incompressible Euler flow in flat and curved spaces. J. Fluid Mech. 825, 412-478 (2017) 
37. Arnold, V.I.: The asymptotic Hopf invariant and its applications. In: Vladimir I. Arnold-Collected Works, pp. 357-375. Springer (1974)

38. Arnold, V.I., Khesin, B.A.: Topological methods in hydrodynamics, vol. 125. Springer, Berlin (1999)

39. Robinson, J.C., Rodrigo, J.L., Sadowski, W.: The Three-Dimensional Navier-Stokes Equations: Classical Theory, vol. 157. Cambridge University Press, Cambridge (2016)

40. Boyer, F., Fabrie, P.: Mathematical Tools for the Study of the Incompressible Navier-Stokes Equations and Related Models. Springer, New York (2013)

41. Leahy, J.-M., Mikulevičius, R.: On degenerate linear stochastic evolution equations driven by jump processes. Stoch. Process. Appl. 125(10), 3748-3784 (2015)

42. Gerencsér, M., Gyöngy, I., Krylov, N.: On the solvability of degenerate stochastic partial differential equations in Sobolev spaces. Stoch. Partial Differ. Equ. Anal. Comput. 3(1), 52-83 (2015)

43. Majda, A.J., Bertozzi, A.L.: Vorticity and Incompressible Flow. Cambridge Texts in Applied Mathematics, vol. 27. Cambridge University Press, Cambridge (2002)

44. Krylov, N.V., Rozovskii, B.L.: Characteristics of degenerating second-order parabolic Itô equations. J. Math. Sci. 32(4), 336-348 (1986)

45. Rozovsky, B.L., Lototsky, S. V.: Stochastic Evolution Systems. Springer International Publishing, Lototsky (2018)

46. Leahy, J.-M., Mikulevičius, R.: On some properties of space inverses of stochastic flows. Stoch. Partial Differ. Equ. Anal. Comput. 3(4), 445-478 (2015)

47. Leahy, J.-M., Mikulevičius, R.: On classical solutions of linear stochastic integro-differential equations. Stoch. Partial Differ. Equ. Anal. Comput. 4(3), 535-591 (2016)

48. Cruzeiro, A.B., Holm, D.D., Ratiu, T.S.: Momentum maps and stochastic Clebsch action principles. Commun. Math. Phys. 357(2), 873-912 (2018)

49. Arnaudon, A., De Castro, A.L., Holm, D.D.: Noise and dissipation on coadjoint orbits. J. Nonlinear Sci. 28(1), 91-145 (2018)

50. Alonso-Orán, D., de León, A.B., Takao, S.: The Burgers' equation with stochastic transport: shock formation, local and global existence of smooth solutions. arXiv preprint arXiv:1808.07821 (2018)

51. Eyink, G.L., Drivas, T.D.: Spontaneous stochasticity and anomalous dissipation for burgers equation. J. Stat. Phys. 158(2), 386-432 (2015)

52. Bernard, D., Gawedzki, K., Kupiainen, A.: Slow modes in passive advection. J. Stat. Phys. 90(3-4), 519-569 (1998)

53. Eyink, G., Vishniac, E., Lalescu, C., Aluie, H., Kanov, K., Bürger, K., Burns, R., Meneveau, C., Szalay, A.: Flux-freezing breakdown in high-conductivity magnetohydrodynamic turbulence. Nature 497(7450), 466 (2013)

54. Lazarian, A., Eyink, G., Vishniac, E., Kowal, G.: Turbulent reconnection and its implications. Philos. Trans. R. Soc. A 373(2041), 20140144 (2015)

55. Lalescu, C.C., Shi, Y.-K., Eyink, G.L., Drivas, T.D., Vishniac, E.T., Lazarian, A.: Inertial-range reconnection in magnetohydrodynamic turbulence and in the solar wind. Phys. Rev. Lett. 115(2), 025001 (2015)

56. Gawȩdzki, K., Vergassola, M.: Phase transition in the passive scalar advection. Physica D 138(1-2), 63-90 (2000)

57. Falkovich, G., Gawȩdzki, K., Vergassola, M.: Particles and fields in fluid turbulence. Rev. Mod. Phys. 73(4), 913 (2001)

58. Eyink, G.L.: Turbulent cascade of circulations. Comptes Rendus Phys. 7(3-4), 449-455 (2006)

59. Jucha, J., Haitao, X., Pumir, A., Bodenschatz, E.: Time-reversal-symmetry breaking in turbulence. Phys. Rev. Lett. 113(5), 054501 (2014)

60. Drivas, T.D.: Turbulent cascade direction and Lagrangian time-asymmetry. J. Nonlinear Sci. 29(1), 65-88 (2019)

61. Holm, D.D, Marsden, J.E.: Momentum maps and measure-valued solutions (peakons, filaments, and sheets) for the epdiff equation. In: The breadth of symplectic and Poisson geometry, pp 203-235. Springer (2005)

62. Crisan, D., Holm, D.D.: Wave breaking for the stochastic Camassa-Holm equation. Physica D 376, 138-143 (2018)

63. Camassa, R., Holm, D.D.: An integrable shallow water equation with peaked solitons. Phys. Rev. Lett. 71(11), 1661 (1993)

64. Bendall, T.M., Cotter, C.J., Holm, D.D.: Perspectives on the formation of peakons in the stochastic Camassa-Holm equation. arXiv preprint arXiv:1910.03018 (2019)

65. Hijón, C., Español, P., Vanden-Eijnden, E., Delgado-Buscalioni, R.: Mori-Zwanzig formalism as a practical computational tool. Faraday Discuss. 144, 301-322 (2010) 
66. Arnold, V.I.: Sur un principe variationnel pour les écoulements stationnaires des liquides parfaits et ses applications aux problemes de stabilité non linéaires. J. Mécanique 5(1), 29 (1966)

67. Arnold, V.I.: On the differential geometry of infinite-dimensional Lie groups and its application to the hydrodynamics of perfect fluids. In: Vladimir I. Arnold-Collected Works, pp. 33-69. Springer (1966)

68. Ebin, D.G., Marsden, J.: Groups of diffeomorphisms and the motion of an incompressible fluid. Ann. Math 92(1), 102-163 (1970)

69. Holm, D.D., Marsden, J.E., Ratiu, T., Weinstein, A.: Nonlinear stability of fluid and plasma equilibria. Phys. Rep. 123(1-2), 1-116 (1985)

70. Marsden, J.E., Weinstein, A.: Comments on the history, theory, and applications of symplectic reduction. In: Quantization of singular symplectic quotients, pp 1-19. Springer (2001)

71. Weinstein, A.: The Geometry of Momentum, Géométrie au XXeme Siecle, Histoire et Horizons, pp. 236-245. Hermann, Paris (2005)

72. Poincaré, H.: Sur une forme nouvelle des équations de la mécanique. CR Acad. Sci 132, 369-371 (1901)

73. Holm, D.D.: Euler-Poincaré dynamics of perfect complex fluids. In: Geometry, Mechanics, and Dynamics, pp. 169-180. Springer (2002)

74. Gay-Balmaz, F., Tronci, C.: The helicity and vorticity of liquid-crystal flows. Proc. R. Soc. A 467(2128), $1197-1213(2010)$

75. Jost, J.: Riemannian Geometry and Geometric Analysis, vol. 42005. Springer, Berlin (2008)

76. Morrey Jr., C.B.: Multiple Integrals in the Calculus of Variations. Springer, Berlin (2009)

77. Richard, S.P.: Foundations of Global Non-linear Analysis. W. A. Benjamin, New York (1968)

Publisher's Note Springer Nature remains neutral with regard to jurisdictional claims in published maps and institutional affiliations. 